\title{
Characterizing Acupuncture Stimuli Using Brain Imaging with fMRI - A Systematic Review and Meta- Analysis of the Literature
}

\section{Citation}

Huang, Wenjing, Daniel Pach, Vitaly Napadow, Kyungmo Park, Xiangyu Long, Jane Neumann, Yumi Maeda, Till Nierhaus, Fanrong Liang, and Claudia M. Witt. 2012. “Characterizing Acupuncture Stimuli Using Brain Imaging with fMRI - A Systematic Review and Meta-Analysis of the Literature." Edited by Ben J. Harrison. PLoS ONE 7 (4) (April 9): e32960. doi:10.1371/ journal.pone.0032960.

\section{Published Version}

doi:10.1371/journal.pone.0032960

\section{Permanent link}

http://nrs.harvard.edu/urn-3:HUL.InstRepos:37140364

\section{Terms of Use}

This article was downloaded from Harvard University's DASH repository, and is made available under the terms and conditions applicable to Other Posted Material, as set forth at http:// nrs.harvard.edu/urn-3:HUL.InstRepos:dash.current.terms-of-use\#LAA

\section{Share Your Story}

The Harvard community has made this article openly available. Please share how this access benefits you. Submit a story. 


\title{
Characterizing Acupuncture Stimuli Using Brain Imaging with fMRI - A Systematic Review and Meta-Analysis of the Literature
}

\author{
Wenjing Huang ${ }^{1,2}$, Daniel Pach ${ }^{1}$, Vitaly Napadow ${ }^{4,5}$, Kyungmo Park ${ }^{6}$, Xiangyu Long ${ }^{8}$, Jane Neumann ${ }^{8,9}$, \\ Yumi Maeda ${ }^{4,5}$, Till Nierhaus ${ }^{7,8}$, Fanrong Liang ${ }^{2}$, Claudia M. Witt ${ }^{1,3 *}$
}

1 Institute for Social Medicine, Epidemiology and Health Economics, Charité University Medical Center, Berlin, Germany, 2 Chengdu University of Traditional Chinese Medicine, Chengdu, China, 3 Center for Integrative Medicine, University of Maryland School of Medicine, Baltimore, Maryland, United States of America, 4 Athinoula A. Martinos Center for Biomedical Imaging, Department of Radiology, Massachusetts General Hospital, Charlestown, Massachusetts, United States of America, $\mathbf{5}$ Department of Radiology, Logan College of Chiropractic, Chesterfield, Missouri, United States of America, 6 Department of Biomedical Engineering, Kyung Hee University, Yongin, Republic of Korea, 7 Berlin Neurolmaging Center and Department Neurology, Charité, Berlin, Germany, 8 Max Planck Institute for Human Cognitive and Brain Sciences, Leipzig, Germany, 9 Leipzig University Medical Center, IFB Adiposity Diseases, Leipzig, Germany

\begin{abstract}
Background: The mechanisms of action underlying acupuncture, including acupuncture point specificity, are not well understood. In the previous decade, an increasing number of studies have applied fMRI to investigate brain response to acupuncture stimulation. Our aim was to provide a systematic overview of acupuncture fMRI research considering the following aspects: 1) differences between verum and sham acupuncture, 2) differences due to various methods of acupuncture manipulation, 3) differences between patients and healthy volunteers, 4) differences between different acupuncture points.

Methodology/Principal Findings: We systematically searched English, Chinese, Korean and Japanese databases for literature published from the earliest available up until September 2009, without any language restrictions. We included all studies using $\mathrm{fMRI}$ to investigate the effect of acupuncture on the human brain (at least one group that received needlebased acupuncture). 779 papers were identified, 149 met the inclusion criteria for the descriptive analysis, and 34 were eligible for the meta-analyses. From a descriptive perspective, multiple studies reported that acupuncture modulates activity within specific brain areas, including somatosensory cortices, limbic system, basal ganglia, brain stem, and cerebellum. Meta-analyses for verum acupuncture stimuli confirmed brain activity within many of the regions mentioned above. Differences between verum and sham acupuncture were noted in brain response in middle cingulate, while some heterogeneity was noted for other regions depending on how such meta-analyses were performed, such as sensorimotor cortices, limbic regions, and cerebellum.

Conclusions: Brain response to acupuncture stimuli encompasses a broad network of regions consistent with not just somatosensory, but also affective and cognitive processing. While the results were heterogeneous, from a descriptive perspective most studies suggest that acupuncture can modulate the activity within specific brain areas, and the evidence based on meta-analyses confirmed some of these results. More high quality studies with more transparent methodology are needed to improve the consistency amongst different studies.
\end{abstract}

Citation: Huang W, Pach D, Napadow V, Park K, Long X, et al. (2012) Characterizing Acupuncture Stimuli Using Brain Imaging with fMRI - A Systematic Review and Meta-Analysis of the Literature. PLoS ONE 7(4): e32960. doi:10.1371/journal.pone.0032960

Editor: Ben J. Harrison, The University of Melbourne, Australia

Received June 14, 2011; Accepted February 8, 2012; Published April 9, 2012

Copyright: (c) 2012 Huang et al. This is an open-access article distributed under the terms of the Creative Commons Attribution License, which permits unrestricted use, distribution, and reproduction in any medium, provided the original author and source are credited.

Funding: Funding provided by Carstens Foundation and Chinese Scholarship Council. The funders had no role in study design, data collection and analysis, decision to publish, or preparation of the manuscript.

Competing Interests: The authors have declared that no competing interests exist.

* E-mail: claudia.witt@charite.de

\section{Introduction}

Acupuncture is a therapy of inserting and manipulating fine filiform needles into specific body locations (acupuncture points) to treat diseases. Acupuncture is an ancient Chinese treatment that has been systematically used for over 2000 years [1]. Currently, acupuncture is used widely all over the world, but its biological mechanism is not well understood. From a neurophysiological aspect acupuncture can be regarded as a complex somatosensory stimulation [2]. Although the clinical effect of acupuncture is generally accepted for certain diagnoses [3], such as knee pain, low back pain etc., there exists controversy regarding the specific effect of acupuncture, especially for the specificity of acupuncture points and meridians. In clinical studies large effects produced by sham acupuncture were observed [4-6].

Interest in investigating acupuncture mechanisms with imaging techniques has been growing since the mid $1990 \mathrm{~s}$ [7,8]. Positron emission tomography (PET), single photon emission computed tomography (SPECT), and magnetic resonance imaging (MRI) 
have been used and, there is also interest in electro-encephalography (EEG). Functional MRI (fMRI), investigating the hemodynamic blood oxygenation level dependent (BOLD) effect, has come to dominate the brain mapping field due to its minimal invasiveness, lack of radiation exposure, excellent spatial resolution and relatively wide availability.

In the previous decade, an increasing number of studies applied fMRI to investigate acupuncture stimulation. The aim of this review was to give a systematic overview about the fMRI research on acupuncture regarding the following four aspects: 1) differences between verum and sham acupuncture, 2) differences due to various methods of acupuncture manipulation, 3) differences between patients and healthy volunteers, 4) differences between different acupuncture points.

\section{Methods}

The search strategy, research questions, inclusion and exclusion criteria and data extraction and analysis were predefined in our protocol. During the study, the database search was extended for the Japanese and Korean databases.

\section{Searching}

We searched the following sources:

1.PubMed (1948-2009.09) 2.EMBASE (1980-2009.09) 3. CNKI (China National Knowledge Infrastructure) (19152009.09) 4.Japanese Ichushi-Web (1983-2009.09) 5.Korean NDSL (National Digital Science Links) (1946-2009.09); KTKP (Korean Traditional Knowledge Portal) (1997-2008)

We searched these databases in the appropriate language using the following MeSH terms and search strategies:

English: 1.fMRI; 2.Functional MRI; 3.MRI, Functional; 4. Magnetic Resonance Imaging, Functional; 5.acupuncture; 6.\#1 or \#2 or \#3 or \#4; 7.\#5 and \#6;

Chinese: 1. 针刺(acupuncture); 2. 磁共振成像(Magnetic Resonance Imaging); 3 .\#1 and \#2

Japanese: 1.1.鍼(acupuncture); 2.機能的磁気共鳴画像法 (Functional Magnetic Resonance Imaging); 3.\#1 and \#2

Korean: 1.기능적 자기공명영상 (fMRI, functional Magnetic Resonance Imaging); 2.침 (acupuncture); 3 .\#1 and \#2

We screened the bibliographies of identified trials and reviewed articles for further potentially relevant publications.

\section{Selection}

In this review we included all studies using fMRI to investigate the effect of acupuncture on the human brain. Each study had to have at least one group, which received an intervention with any type of needle-based acupuncture. We included trials on healthy volunteers as well as patients and all types of needle acupuncture were accepted. There were no language restrictions and no limitations on outcome measures. Reviews, editorials and trials on animals were excluded.

The available abstracts of all identified references were screened and we excluded all citations that clearly did not fit the inclusion criteria. Full copies of all remaining articles and those references without available abstracts were obtained. Subsequently the three researchers (WJH: Pubmed, Embase and CNKI, KP: Korean databases, YM: Japanese databases) screened the full texts and assessed whether these trials met the inclusion criteria.

In the meta-analysis, we included studies investigating only verum acupuncture or both verum and sham acupuncture by fMRI using whole brain acquisition. Studies were excluded if 1) the number of study participants was less than five; 2) results were not reported as 3-dimensional coordinates in standard stereotactic space; 3) only the results from regions of interest (ROI) were reported or 4) only single subject data instead of group data were reported.

\section{Data extraction and analysis}

The three researchers (WJH: Pubmed, Embase and CNKI, KP: Korean databases, YM: Japanese databases) extracted the data for all descriptive information from the publications, namely published journals, language, study place, study type, subjects, handedness, objective, interventions, control groups, block-design, fMRI device type, software for fMRI data analysis, sample size, and results. The extracted data were discussed with three supervisors (CW, DP and VN). Any inconsistencies were discussed and reconsidered until consensus was reached.

Results were structured according to the four research questions. Studies that matched multiple research questions were displayed more than once, but only with the part of the study relevant to the respective research question.

Furthermore, one figure for different acupuncture points from publications in Talairach coordinates was generated by one author (XYL) using Analysis of Functional NeuroImages (AFNI, http:// afni.nimh.nih.gov) and MRIcron software (http://www.cabiatl. $\mathrm{com} / \mathrm{mricro})$. The anatomical image was generated using MRIcron software.

The meta-analyses were conducted (JN, XYL, WJH) in Talairach space, using the activation likelihood estimation technique (ALE) implemented in GingerALE 2.1.1 software [911]. This technique assesses the convergence between activation foci from different experiments. Prior to the analysis, coordinates reported in MNI (Montreal Neurological Institute) space were converted to Talairach anatomical space using the Lancaster transform [12]. For each experiment, every reported activation maximum was modeled by a 3-dimensional Gaussian probability distribution centered at the given coordinate. The width of the Gaussian probability distribution was determined individually for each experiment based on empirical estimates of between-subject variability, taking into account the number of subjects in each experiment [9]. Voxel-wise ALE scores were calculated from the union of the Gaussian probability distributions within and across experiments. In a random effects analysis, ALE scores were tested against a null hypothesis of random distribution across the brain, thereby identifying those regions where empirical ALE values were higher than could be expected by chance. Resulting ALE maps were thresholded at $\mathrm{p}<0.05$ (corrected for multiple comparisons by False Discovery Rate). The minimum cluster volume was chosen to exceed the number of voxels corresponding to $5 \%$ possible false positives. The contrast studies analysis (subtraction analysis which compares two ALE maps) was performed with randomization testing with 10,000 permutations. As there exists no correction for multiple comparison with this approach, the threshold was set at $\mathrm{p}<0.05$ (uncorrected) with a min. cluster size $=200 \mathrm{~mm}^{3}[13]$.

ALE maps were computed for the following statistical comparisons. From all studies included in the meta-analysis: 1a) greater activation of verum acupuncture points compared to baseline (verum $>$ rest), lb) greater deactivation of verum acupuncture points compared to baseline (rest>verum). From the studies which provided direct contrasts between verum and sham acupuncture: 2a) greater activation from verum than sham acupuncture (or greater deactivation for sham, i.e. verum $>$ sham), $2 \mathrm{~b}$ ) greater deactivation from verum than sham acupuncture (or greater activation for sham, i.e. sham $>$ verum). From the studies which had both verum and sham acupuncture groups: $3 \mathrm{a})$ greater activation of verum acupuncture points than baseline (verum $>$ 
rest), 3b) greater deactivation of verum acupuncture points than baseline (rest>verum), 3c) greater activation of sham acupuncture points than baseline (sham $>$ rest), 3d) greater deactivation of sham acupuncture points than baseline (rest $>$ sham), 3e) comparison ALE map of greater activation of verum than sham acupuncture relative to rest ("verum>rest" - "sham $>$ rest"), 3f) comparison ALE map of greater deactivation of verum than sham acupuncture relative to rest ("rest>verum" - "rest>sham").

\section{Results}

\section{Study characteristics}

The 149 studies were published between 1999 and 2009 (trial flow see Figure 1), Figure 2 shows the number of publications per year in corresponding countries in the last 11 years. Most of the studies were performed in China, US and Korea and predominantly published in Chinese and English (50.3\% Chinese, 38.9\% English, 9.4\% Korean, $0.7 \%$ German and $0.7 \%$ Japanese). The median number of subjects per study was 17 (min. 1 to max. 67), and the total number of all studies included 2469 subjects. 24 studies reported parallel group randomized trials. 128 studies were on healthy volunteers, 13 studies on patients, 8 studies on the comparison of patients and healthy volunteers. Most of the trials applied a block design for fMRI data acquisition, with a time range for each block of $8 \mathrm{sec}$ to $6 \mathrm{~min}$, and the number of blocks ranged from one to 12 blocks. 105 studies included right-handed subjects while only 3 studies included also left-handed subjects. 34 studies were included in the meta-analyses.

\section{Descriptive findings of differences between verum and sham acupuncture}

51 publications explored four kinds of sham acupuncture including a) a placebo needle (Streitberger needle [14]: with a blunt tip, which when it touches the skin causes a pricking sensation for the patient, simulating the puncturing of the skin. The needle moves inside the handle, and appears to be shortened.); b) needling at non-acupuncture points in close proximity to acupuncture points; c) needling at non-acupuncture points distant to acupuncture points; d) cutaneous stimulation at the same acupuncture points or sham point/area (Table S1). Two of the studies $[15,16]$ are referenced more than once in the table because of the different sham acupuncture methods evaluated in these studies. The studies included mainly healthy volunteers, but four publications [17-20] included patients with Parkinson's disease or stroke.

A placebo needle: Streitberger Needle. The four studies which compared verum acupuncture with the Streitberger Needle were all from the US and showed heterogeneous results $[16,19,21,22]$. Yoo et al. [16] found more activation associated
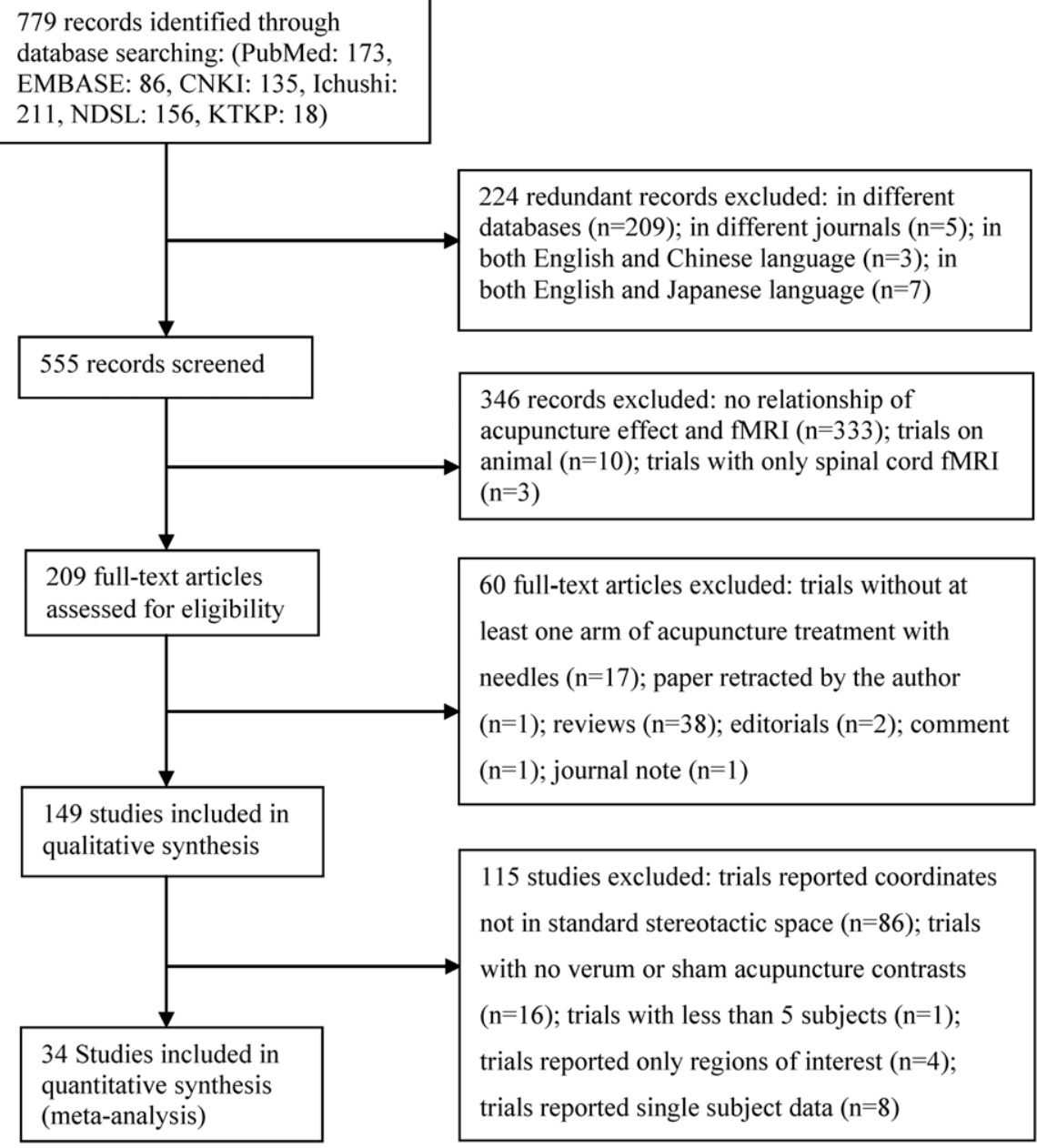

Figure 1. Flow of information through the different phases of the systematic review. doi:10.1371/journal.pone.0032960.g001 


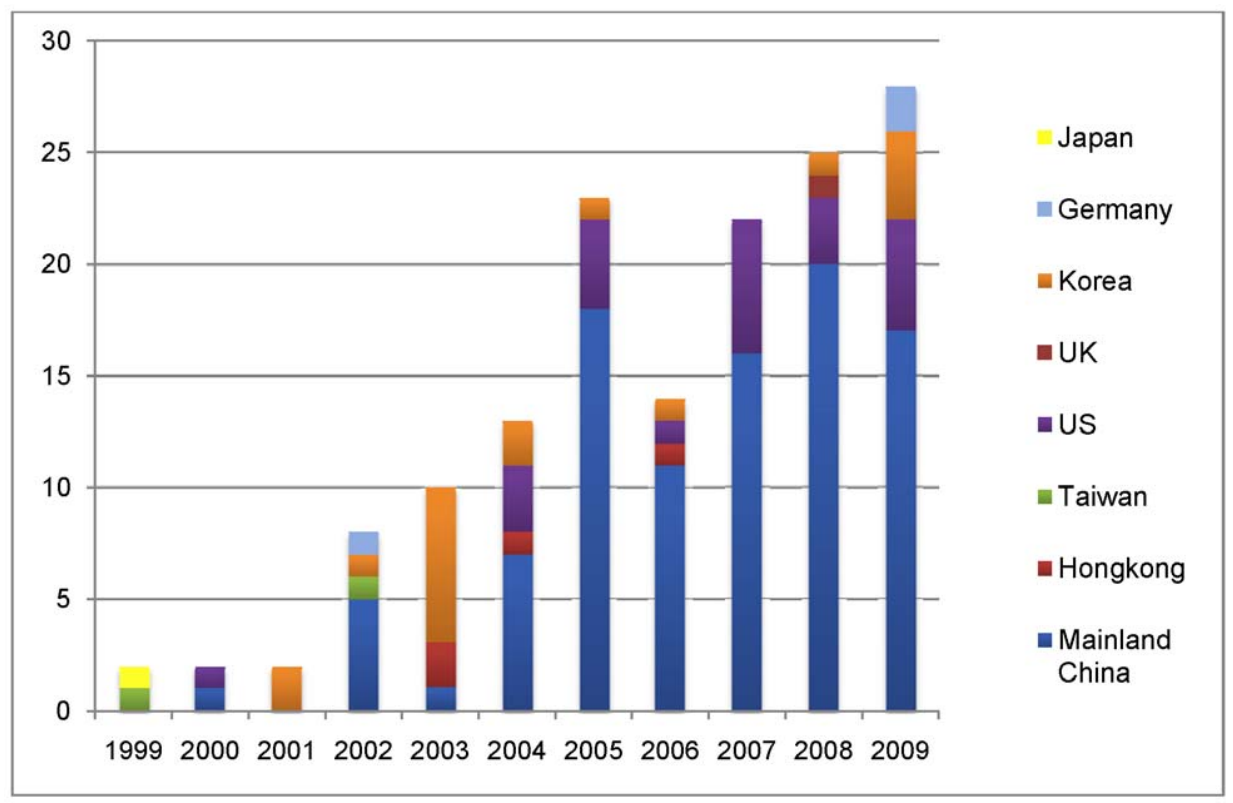

Figure 2. Number of publications on acupuncture and fMRI identified in the last 11 years. doi:10.1371/journal.pone.0032960.g002

with verum acupuncture in the somatosensory areas and motor areas. Dougherty et al. [21] reported that acupuncture produced more activation in the medial orbitofrontal cortex and more deactivation in brainstem and insula, while the Streitberger needle showed higher activation in the language area (Wernicke), pons, operculum and insula. According to Deng et al. [22] verum acupuncture resulted in more activation in insula and operculum compared to the Streitberger needle placed at a non-acupuncture point. A study with stroke patients [19] (scan during passive finger movement pre and -post 10 weeks treatment of verum acupuncture or the Streitberger placebo needle) showed a trend toward a greater maximum activation change in the motor cortical area for the verum acupuncture group.

Acupuncture at non-acupuncture points in close proximity to acupuncture points. Two third (64\%) [15,2337] of 25 studies showed that acupuncture treatments were associated with more activation, mainly in the somatosensory areas, motor areas, basal ganglia, cerebellum, limbic system and higher cognitive areas (e.g. prefrontal cortex). Three studies $[28,37,38]$ showed also more deactivations in the limbic system in response to acupuncture. In contrast, one study [39] found greater activation in the supplementary motor area in response to sham acupuncture. Five other studies [40-44] found no significant difference between verum and sham acupuncture. One experiment was analyzed twice $[45,46]$ and came to different results.

Acupuncture at non-acupuncture points distant to acupuncture points. Of six studies, two studies $[47,48]$ showed no differences between verum and sham acupuncture. Four studies [49-52] showed more activation associated with acupuncture in the somatosensory areas, brainstem, basal ganglia, higher cognitive areas and part of the limbic system (hypothalamus, nucleus accumbens), and one study [52] showed more activation associated with sham acupuncture in the motor area and operculum. Verum acupuncture showed also more deactivation in part of the limbic system (amygdala, hippocampus, cingulate gyrus/cortex) [47,52]. In addition, Napadow et al. [51] found that both verum and sham acupuncture showed linearly decreasing activation over repeated stimulus blocks in the sensorimotor areas, while verum acupuncture produced bimodal activity in a limbic midbrain region - activation in early blocks, but deactivation in later stimulus blocks.

Cutaneous stimulation at the same acupuncture point or sham point/area. There are 18 studies (15 on healthy volunteers). Only one study [16] on healthy volunteers found greater activation in the somatosensory area during verum acupuncture, whereas in four studies [53-56] somatosensory activation was greater with cutaneous stimulation. For motor areas and higher cognitive areas, five studies $[15,16,55,57,58]$ showed that acupuncture was associated with more activation. For brainstem, basal ganglia, cerebellum and limbic system the results were complex or contradictory: in the basal ganglia, brainstem and cerebellum, two studies [53,59] found that acupuncture was associated with more deactivation while three other studies $[15,57,60]$ found acupuncture associated with more activation; thalamus and insula $[15,16,54,58]$ were activated more while hypothalamus, hippocampus, amygdala and temporal pole $[53,54,58,59]$ were deactivated more by acupuncture. In addition, when eliciting deqi, Hui et al. [53] found extensive deactivation in the cerebrum, brainstem and cerebellum, while eliciting deqi mixed with pain, activation was the predominant pattern. Five Chinese studies [61-65] found almost no significant differences between verum and sham, though two of them found greater activation intensity in the cerebellum or parietal lobe for verum acupuncture $[61,62]$. Among the three publications on patients, Schockert et al. [20] found more activation in the motor area on stroke patients during acupuncture while $\mathrm{Li}$ et al. [17] found more activation in the somatosensory and motor areas with a control, brushing stimulation on stroke patients. In patients with Parkinson's disease Chae et al. [18] showed that acupuncture was associated with more activation than covert cutaneous stimulation in the motor area, basal ganglia, visual and higher cognitive area; and more activation in the motor, visual, higher cognitive areas and limbic system, compared to overt cutaneous stimulation. 
Descriptive findings of differences due to various methods of acupuncture manipulation

Manipulation methods can differ in the depth of needling, forms of needle stimulation (e.g. manual versus electrical), intensity of stimulation, and stimulus timing parameters (e.g. duration, frequency, etc.). Here, we summarized the results from those studies comparing different methods of manipulation at acupuncture points in healthy volunteers (see Table 1). Two of the studies $[58,66]$ are displayed more than once in the table as they explored multiple comparisons.

Comparison of different needling depths. Of four studies, two studies $[67,68]$ found no significant difference between deep and superficial needling. Whereas Zhang et al. [25] found more activation in almost all brain areas from deep needling and $\mathrm{Wu}$ et al. [52] found more activation from superficial needling in the somatosensory area, motor area and language areas (Broca and Wernicke areas), and from deep needling more deactivation in the limbic system.

Comparison of electro-acupuncture vs. manual acupuncture. Overall, the results of three studies showed that electro-acupuncture tends to produce more activation and less deactivation compared to manual acupuncture. Regarding brain activations, two studies [58,69] found more activation associated with electro-acupuncture in somatosensory areas, motor area, brainstem, cingulate or insula and one study [66] found no significant difference. Regarding brain deactivations, two studies $[66,69]$ showed manual acupuncture was associated with more deactivation in the limbic system [69], cuneus [66], transverse temporal gyrus [66] or middle frontal gyrus [66], yet two studies $[58,69]$ also showed more deactivation from electro-acupuncture in the septal area or precuneus.

Comparison of different frequencies of electroacupuncture stimulation. Two studies compared different electro-acupuncture frequencies. Napadow et al. [58] found that the brainstem was more activated at $2 \mathrm{~Hz}$ than at $100 \mathrm{~Hz}$. But Li et al. [66] found no significant difference between $2 \mathrm{~Hz}$ and $20 \mathrm{~Hz}$.

Comparison of different intensities of manual acupuncture stimulation. Of six studies one study [70] observed that a longer duration of manipulation induced more activation in the inferior frontal, temporal, parietal gyrus, occipital lobe, cerebellum or temporal pole and more deactivation in the prefrontal cortex, orbital gyrus or pons than shorter manipulation. Four studies [42,71-73] found more activation in the somatosensory areas, limbic system, visual, language areas or higher cognitive areas in response to stimulation compared to no stimulation. The last study [74] showed that stimulation which induced deqi by maximum manipulation was associated with more activation in the postcentral gyrus and the limbic system than stimulation that didn't induce deqi with minimum manipulation.

\section{Descriptive findings of differences between patients and healthy volunteers}

All seven studies comparing healthy volunteers with patients showed that patients responded differently (See Table 2). According to Wang et al. [75] the frontal lobe was activated in stroke patients while motor areas were activated in healthy volunteers. Fu et al. [76] found patients with Alzheimer's disease had more activation in the cingulate gyrus and cerebellum. Liu et al. [77] found more robust activation in the hypothalamus in heroin addicts. Wu et al. [78] found deactivation in primary motor cortex (M1), parahippocampal gyrus, and higher cognitive areas and more activation in the cuneus and the insula in children with spastic cerebral palsy but not in healthy children. Conversely, more activation in caudate nucleus, thalamus and cerebellum was found in healthy children. Napadow et al. [79] compared patients with carpal tunnel syndrome (CTS) before and after five weeks' acupuncture to healthy volunteers receiving no treatment. Following acupuncture, a significant decrease in the activation area was found in contralateral primary somatosensory cortex (SI) and M1 in the CTS patients, as well as, increased separation between digit 3 and digit 2 cortical representations in SI, suggesting acupuncture-induced neuroplasticity. In addition, Napadow et al. compared manual acupuncture to cutaneous stimulation on both CTS patients and healthy volunteers. They found that CTS patients responded to verum acupuncture with less deactivation in the amygdala and greater activation in the lateral hypothalamic area [80], compared to healthy subjects. Moreover, CTS patients responded to sham acupuncture with greater activation in the somatosensory areas, cognitive and affective areas. Li et al. [17] found that stroke patients had more activation in the SI than healthy volunteers when both groups underwent both verum and sham acupuncture.

\section{Descriptive findings of differences between different acupuncture points}

The data on acupuncture point specific changes in brain activation and deactivation are shown in Table S2, originating from 76 publications $[15,16,22,24,27,31,33,42,45-47,51-$ 53,55,57-59,61,63,64,67,69,81-107] [29,34,37,39,40,43,73,108126] addressing 37 acupuncture points. Acupuncture points along the 12 regular meridians and one extra meridian (Du meridian) were assessed. The data showed changes in brain activity for each individual acupuncture point from respective publications. The most studied points were LI4, ST36, PC6, LR3 and GB34. These points have a wide clinical applicability and are frequently used in clinical practice. Overall the data showed that acupuncture stimulation mainly influenced the brain activity of the somatosensory areas, motor areas, auditory areas, visual areas, cerebellum, the limbic system and higher cognitive areas.

Furthermore, we generated on a descriptive level map (Figure 3) of 18 acupuncture points from 46 publications, which reported pre-post data on Talairach coordinates. These 18 points were located along 9 meridians. The brain maps of each acupuncture point differ considerably from each other. However, the acupuncture points on the same meridian showed some similarities among the activation/deactivation pattern. For example, the points on the stomach meridian showed activation in the supramarginal gyrus and deactivation in the posterior cingulate, hippocampus, and parahippocampus. In addition, the vision related points GB37 and UB60 showed deactivation in the visual areas such as the cuneus.

\section{Descriptive findings of other comparisons and results}

Besides our four main research questions, there are more research findings worth mentioning: comparisons between acupuncture and other stimulations; comparisons of acupuncture under different consciousness states; acupuncture at different time points; acupuncture at group of points; acupuncture effect correlated to expectation. Moreover, resting state functional connectivity was also investigated in several recently published papers.

Acupuncture vs. visual stimulation. Of four studies, Bai et al. [127] compared the stimulation phase and the resting phase of acupuncture stimulation and visual stimulation and found the BOLD signal returned to near-baseline values shortly after the visual stimulus, but for acupuncture stimulation the resting phase activities might be even higher than that of the stimulation phases. 


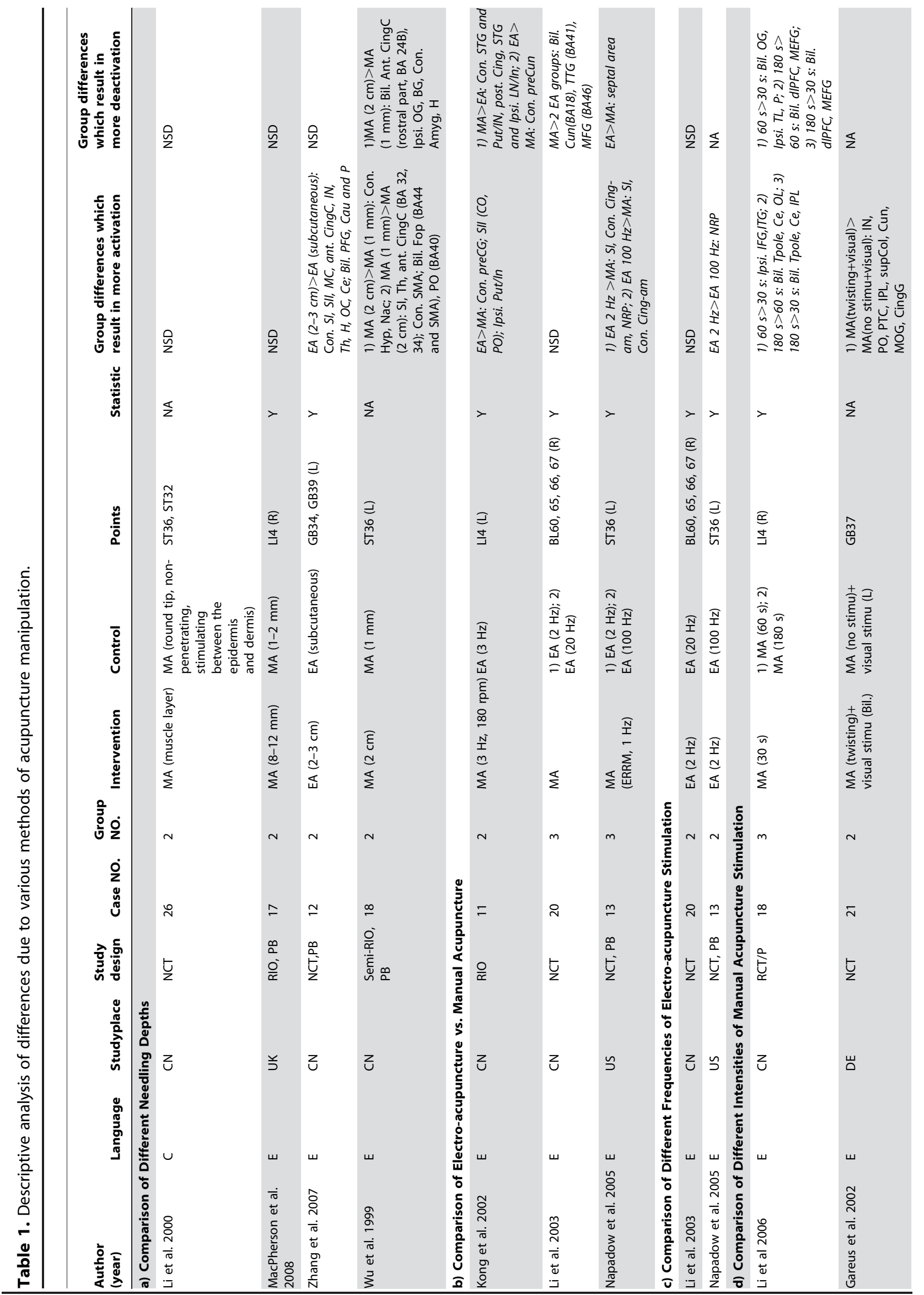




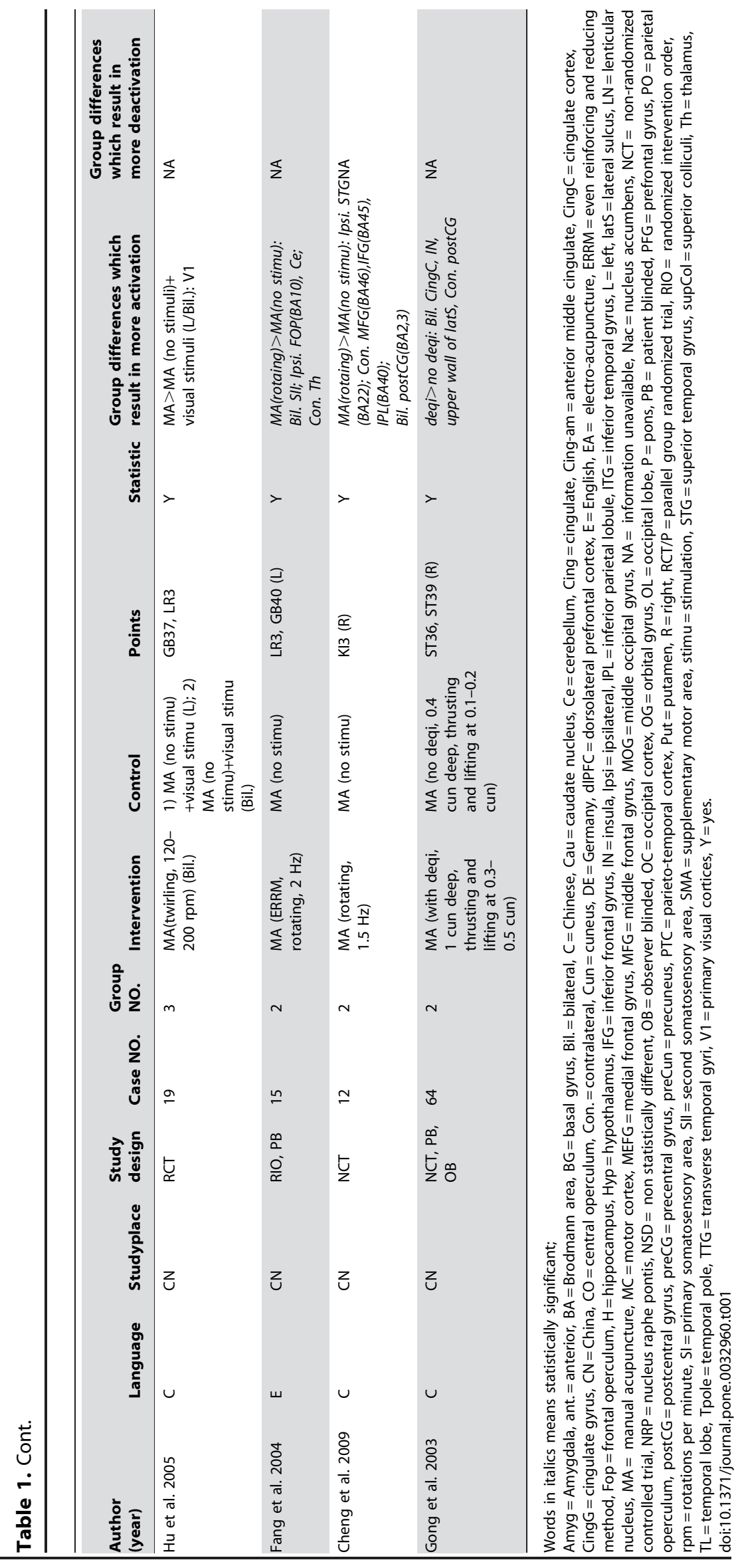




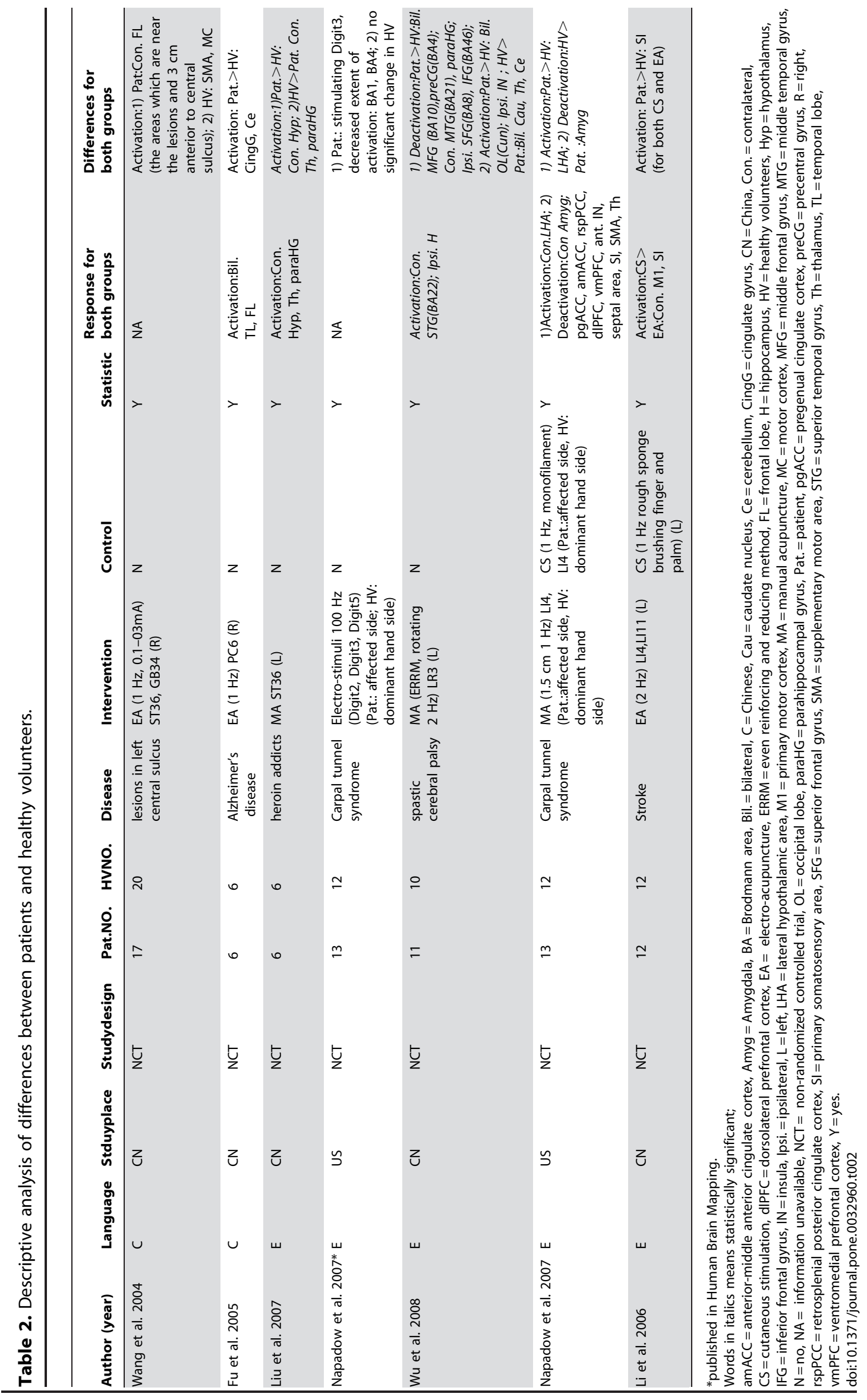




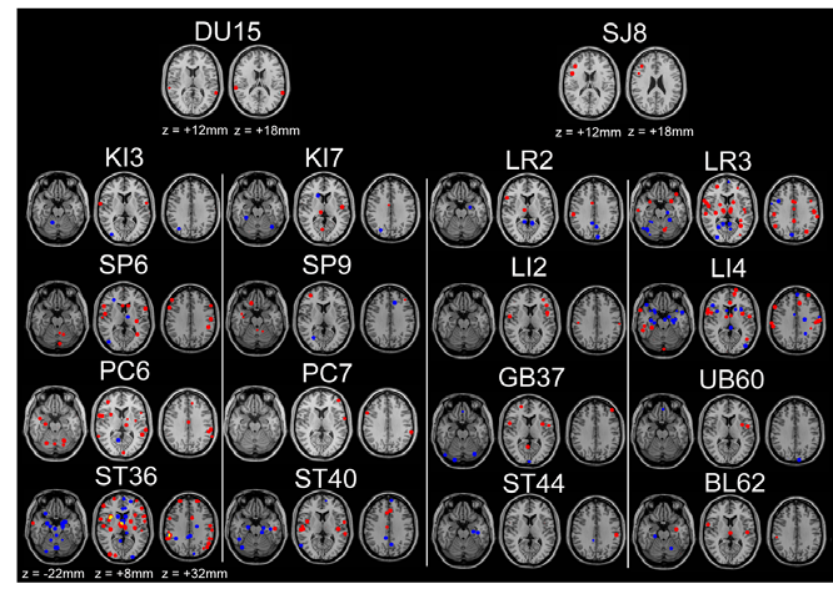

Figure 3. Map of brain response to $\mathbf{1 8}$ different acupuncture points. Red: activation; Blue: deactivation; Yellow: overlap. doi:10.1371/journal.pone.0032960.g003

$\mathrm{Hu}$ et al. [71] and Gareus et al. [72] had contradictory results. Surprisingly, $\mathrm{Hu}$ et al. [71] reported no significant activation in the visual cortex during visual stimulation but from acupuncture stimulation, whereas Gareus et al. [72] found no activation in the visual cortex during acupuncture stimulation, and activation from visual stimulation. Li et al. [66] found both visual stimulation and acupuncture could activate the visual cortex.

Acupuncture vs. word generation paradigm. One study from $\mathrm{Li}$ et al. [29] found acupuncture at language specific acupuncture points SJ8 and Du15 did not activate the typical language areas in the left inferior frontal cortex which were activated during a word-generation task.

Acupuncture vs. finger tapping. Of three studies, both Kong et al. [39] and $\mathrm{Hu}$ et al. [81] found finger-tapping task can produce more reliable fMRI signal changes than that evoked by electro-acupuncture stimulation. However, Wang et al. [128] found no significant difference between electro-acupuncture at ST36, GB34 and a finger-tapping task.

Acupuncture in different states of consciousness (awake or anesthetized). One study from Wang et al. [129] compared healthy subjects who underwent acupuncture at ST36 in two different consciousness states. The result showed activation in the awake state was greater than under anesthetic in the somatosensory area, the limbic system and basal ganglia.

Acupuncture at different time points. One study from Zeng et al. [130] compared acupuncture at KI3 and KI7 at two different time points - "open point time" and "closed point time" (the open or closed time point is determined by the Chinese medicine theory "Zi-Wu-Liu-Zhu"-the body's Qi and blood circulation schedule; acupuncture at the open point time results in maximum clinical effect and vice versa) [1]. The result showed acupuncture at "open point time" was associated with more deactivation in the frontal lobe, temporal lobe, cingulate cortex and cerebellum than acupuncture at the "closed point time".

Acupuncture of group of points. In 29 papers [25,35,38,44,49,62,65,68,71,74,75,93,100,109,130-144] more than one acupuncture point was stimulated simultaneously. Of these groups of points, some were functional related, some were on the same meridian, some had close locations for electric stimulation, few were real acupuncture clinical formula. 15 of these 29 papers were included among our first four main questions. Overall the results of these studies were very heterogeneous and only three studies $[93,109,136]$ reported an interaction effect between acupuncture points.

Acupuncture effect correlated to expectations. Three studies by Kong et al. [145-147] applied an expectancy model, and found positive expectation can increase acupuncture analgesia based on the objective fMRI signal changes in response to noxious stimuli. The study indicated that different mechanisms exist between acupuncture analgesia and expectancy evoked placebo analgesia. For the verum acupuncture group, there were only a few small differences (in primary motor cortex and middle frontal gyrus) between the high expectancy side and low expectancy side. However, for the sham acupuncture group, more differences were observed in contralateral operculum, ipsilateral insula, inferior frontal gyrus, medial frontal gyrus and superior frontal gyrus. So this result suggested expectancy might involve distinct mechanisms between verum acupuncture and sham acupuncture.

Functional connectivity modulated by acupuncture. Eight studies investigated functional connectivity of resting state. One of the first such studies (Dhond et al. study [57]) found that verum acupuncture, but not monofilament tapping increased resting state connectivity of the default mode network (DMN) to pain, affective and memory related regions of the brain. Verum acupuncture also increased sensorimotor network (SMN) connectivity to pain-related brain regions. Zhang et al. [148] and Bai et al. [149] found that acupuncture stimulation may induce the modulation of the "acupuncture-related" network, represented by significant changes of functional connectivity in several regions of the brain, such as the bilateral frontal gyrus, bilateral temporal gyrus, inferior parietal lobe, middle occipital gyrus, pre- and postcentral gyrus, anterior cingulate cortex (ACG), parahippocampus, insula, tonsil, pyramis, culmen, precuneus and cuneus. Qin et al. [150,151] identified an amygdala-related network during the resting state both after verum and penetrating sham acupuncture at a nearby point. Compared to sham, verum acupuncture increased the connectivity between the amygdala, the PAG (periaqueductal gray) and the insula, and decreased the connectivity between the amygdala with the middle frontal cortex, the postcentral gyrus and the posterior cingulate cortex (PCG). Zhang et al. [119] compared the visual related functional networks between pre- and post- electro-acupuncture on the visual-related point GB37 and the non-visual related point KI8 and described a positive correlation between the pre-post resting states in visual networks for the GB37 group while an anti-correlation for the KI8 group. Liu et al. [152] found a similar result when comparing electro-acupuncture at GB37 and KI8. In addition, in a later study Liu et al. [153] reported that the DMN could be modulated after electro-acupuncture at the three acupuncture points (GB37, BL60 and KI8) and at a nearby sham point. As for intrinsic connectivity, the PCG and precuneus strongly interacted with other nodes during the pre- and post-stimulation states. The correlation was interrupted between the PCG/precuneus and the ACG. The orbital prefrontal cortex negatively interacted with the left medial temporal cortex only at the acupuncture points.

\section{Results from the ALE meta-analysis}

A total of 34 studies were eligible for the inclusion criteria for the ALE meta-analyses (Table 3). A total of 10 meta-analyses were performed.

The meta-analysis for verum acupuncture stimuli on greater activation of verum acupuncture points compared to baseline (1a, verum $>$ rest) included 36 experiments, 377 subjects and 470 foci. The result showed significant convergence in the supramarginal gyrus, secondary somatosensory cortex (SII), pre-supplementary 


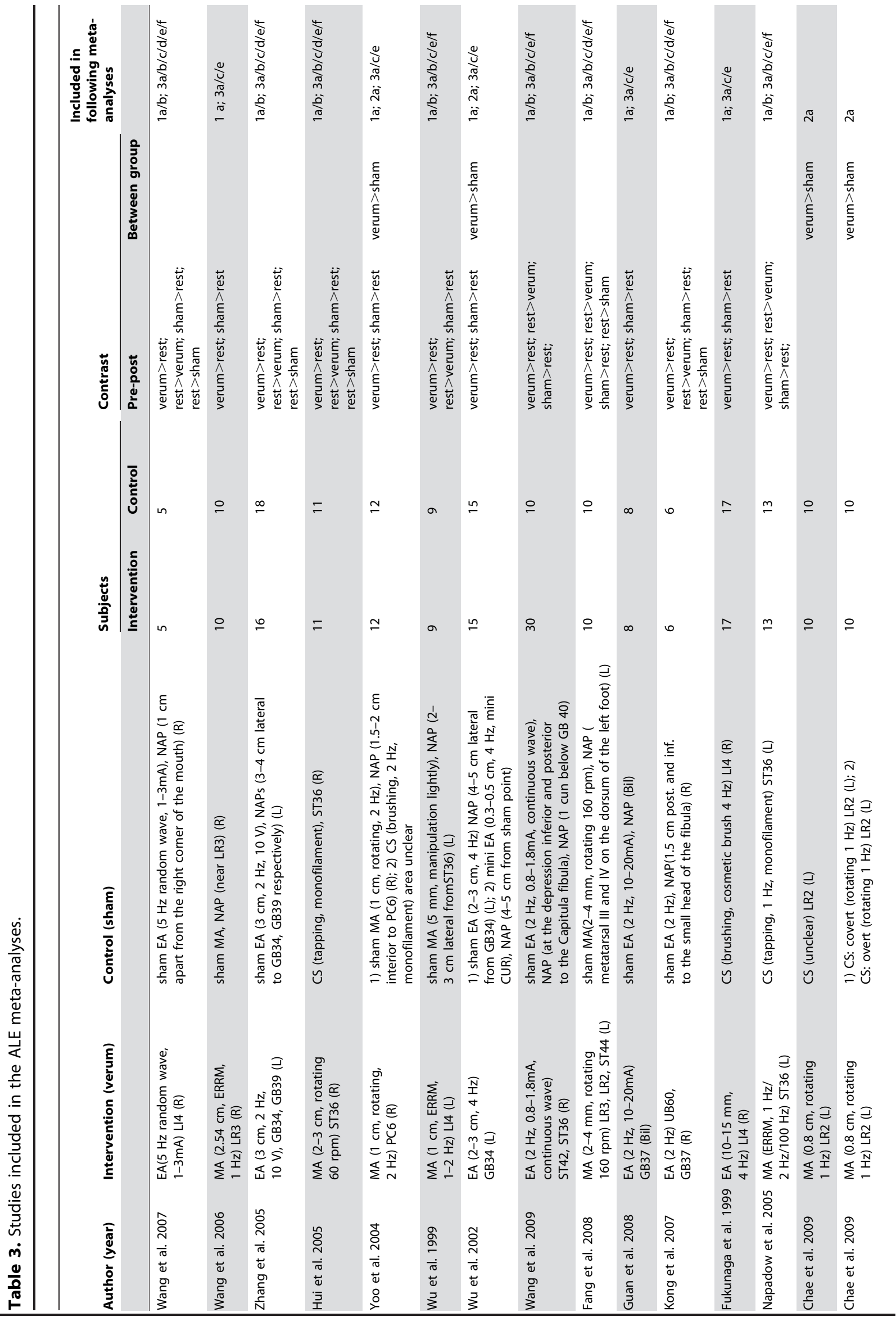




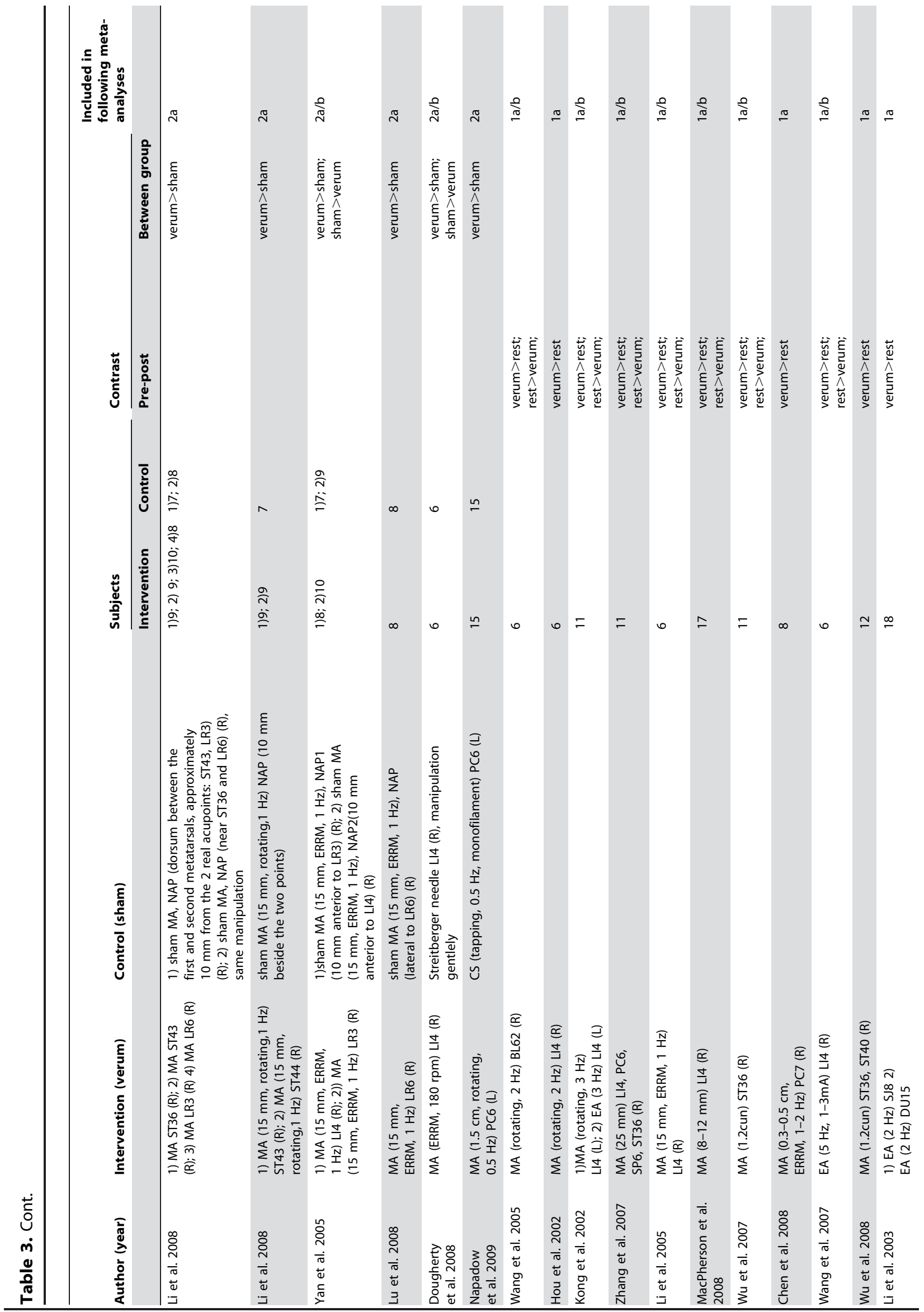




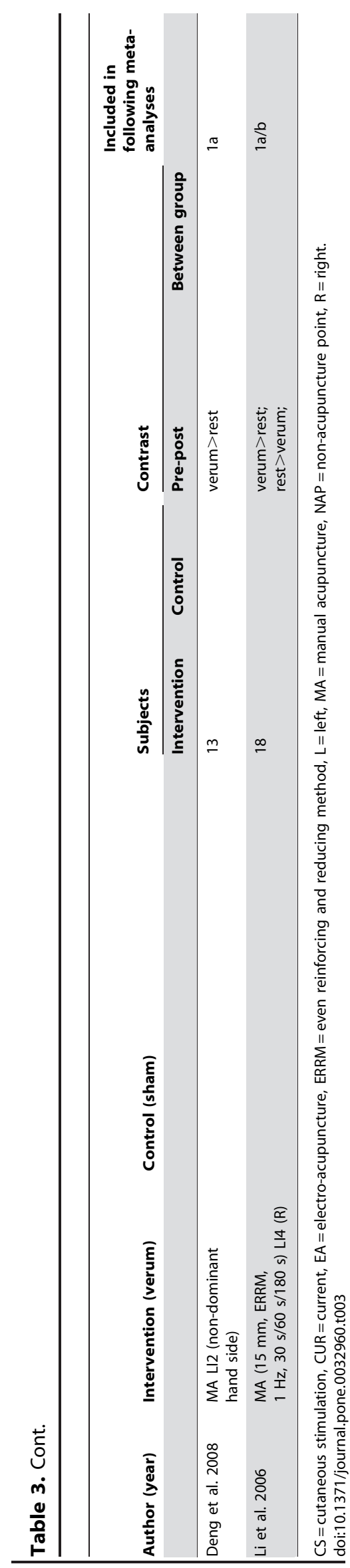

motor area (pre-SMA), middle cigulate gyrus, insula, thalamus and precentral gyrus. The meta-analysis for greater deactivation of verum acupuncture points compared to baseline ( $1 \mathrm{~b}$, rest $>$ verum) included 22 experiments, 219 subjects and 265 foci and the result revealed significant convergence in the subgenual anterior cingulate, subgenual cortex, amygdala/hippocampal formation, ventromedial prefrontal cortex (vmPFG), nucleus accumbens, and PCC (Table 4, Figure 4A).

For the direct contrast of verum and sham acupuncture on greater activation from verum than sham acupuncture or greater deactivation for sham acupuncture $(2 \mathrm{a}$, verum $>$ sham $)$ we included in the meta-analysis 17 experiments, 156 subjects and 171 foci, resulting in significant convergence in fusiform gyrus, cerebellum, SI and middle cingulate gyrus. Whereas, on greater deactivation from verum than sham acupuncture or greater activation for sham ( $2 \mathrm{~b}$, sham $>$ verum, 21 subjects, 3 experiments and 27 foci) the result showed significant convergence in supramarginal gyrus, superior temporal gyrus and cuneus (Table 5, Figure 4B).

The Subtraction analysis for verum versus sham acupuncture included in the first step analyses $3 \mathrm{a}-\mathrm{d}$ for the pre-post contrast on verum or sham acupuncture compared to baseline (Table 5, Figure 4C). The analysis of greater activation of verum acupuncture than baseline ( $3 \mathrm{a}$, verum $>$ rest) included 234 subjects, 20 experiments and 305 foci and revealed significant convergence in middle cingulate gyrus, pre-SMA, superior temporal gyrus, supramarginal gyrus, SII, thalamus and insula. The analysis of greater deactivation of verum acupuncture compared to baseline (3b, rest $>$ verum, 172 subjects, 15 experiments and 222 foci) came to the following significant convergence: subgenual anterior cingulate, amygdala/hippocampal formation, vmPFC and PCG. Comparing results on greater activation of sham acupuncture points than baseline (3c, sham $>$ rest) from 164 subjects, 15 experiments and 200 foci, showed significant convergence in cerebellum, supramarginal gyrus, superior temporal gyrus and thalamus. Including data on greater deactivation of sham acupuncture points compared to baseline ( $3 \mathrm{~d}$, rest $>$ sham) from 50 subjects, 5 experiments and 52 foci, resulted in significant convergence in pregenual anterior cingulate, subgenual cortex and parahippocampal gyrus.

Finally, in the contrast (subtraction) comparing the betweengroup differences for verum and sham acupuncture, significant differences between "verum $>$ rest" and "sham $>$ rest" (3e) as well as between "rest $>$ verum" and "rest $>$ sham" (3f) were identified. The subtraction analysis for "verum $>$ rest" - "sham $>$ rest" showed convergent activations in pre-SMA, middle cingulate gyrus, claustrum, insula, supramarginal gyrus, SII and dorsolateral prefrontal cortex (dlPFG). The subtraction analysis for "rest> verum" - "rest $>$ sham" revealed convergence in amygdala/ hippocampal formation (Table 5, Figure 4D).

\section{Discussion}

Overall the results indicate that studies on acupuncture neuroimaging are very heterogeneous in terms of the study question, methodology and quality, this is the case in the descriptive analysis as well as in the meta-analysis.

From the descriptive view on the data it seems that compared to sham, verum acupuncture tended to be associated with more activation in the basal ganglia, brain stem, cerebellum, and insula and more deactivation was seen in the so-called "default mode network" and limbic brain areas, such as the amygdala and the hippocampus. In addition, a trend for more robust brain activation with greater intensity of acupuncture stimulation seems to be there. However, electro-acupuncture at low frequency also 
Table 4. Clusters showing significant convergence for verum acupuncture points (FDR pN corrected at the cluster level, $p<0.05$ ) from ALE meta-analyses.

\begin{tabular}{|c|c|c|c|c|c|c|}
\hline \multirow[t]{2}{*}{ Brain region } & \multirow[t]{2}{*}{ BA } & \multicolumn{3}{|c|}{ Talairach coordinates } & \multirow[t]{2}{*}{ ALE value } & \multirow[t]{2}{*}{ Volume $\left(\mathrm{mm}^{3}\right)$} \\
\hline & & $\mathbf{x}$ & $\mathbf{y}$ & $\mathbf{z}$ & & \\
\hline \multicolumn{7}{|l|}{ Verum >rest (1a) } \\
\hline \multirow[t]{2}{*}{ Supramaginal gyrus/insula/SII } & 40 & 54 & -26 & 24 & 0.0460 & 15440 \\
\hline & 40 & -54 & -24 & 20 & 0.0565 & 8072 \\
\hline Pre-supplementary motor area/middle cingulate & 6 & -2 & 6 & 48 & 0.0318 & 9576 \\
\hline Thalamus & & 08 & -16 & 8 & 0.0323 & 3776 \\
\hline Precentral gyrus & 44 & -46 & -2 & 8 & 0.0259 & 3696 \\
\hline \multicolumn{7}{|l|}{ Rest>verum (1b) } \\
\hline Anterior cingulate & 32 & 0 & 34 & -8 & 0.0406 & 6032 \\
\hline Subgenual cortex & 25 & 2 & 8 & -4 & 0.0202 & 1304 \\
\hline Amygdala/hippocampal formation & & -28 & -8 & -24 & 0.0253 & 3240 \\
\hline Ventromedial prefrontal cortex & 10 & -2 & 60 & 10 & 0.0261 & 1728 \\
\hline Posterior cingulate & 31 & -6 & -56 & 22 & 0.0188 & 1120 \\
\hline
\end{tabular}

tended to activate a broader range of brain areas than electroacupuncture at high frequencies. Furthermore, it looks like that patients responded to acupuncture stimulation with a more robust fMRI response compared to healthy volunteers. Acupuncture at different acupuncture points showed in the studies both similarities and differences between points. Finally, studies also suggested that acupuncture modulated the resting state connectivity within several noted networks including the default mode network, sensorimotor network, and amygdala-related network etc.

From the meta-analyses focusing only on brain response to verum acupuncture stimuli, activation was noted in supramarginal gyrus, SII, pre-SMA, middle cigulate gyrus, insula, thalamus and precentral gyrus, while deactivation was noted in pregenual anterior cingulate, subgenual cortex, amygdala/hippocampal formation, vmPFC, nucleus accumbens and PCC. Acupuncture specific effects were noted by meta-analyses of differences between verum and sham, which showed greater response in middle cingulate for verum compared to sham acupuncture. However, the results were variant within the different meta-analyses. The metaanalyses of direct contrast between verum and sham showed significant convergence for "verum $>$ sham" in fusiform gyrus, cerebellum and SI, while for "sham > verum" in superior temporal gyrus, supramarginal gyrus and cuneus. Whereas, the subtraction meta-analyses of group-derived contrast showed greater activation from verum in pre-SMA, claustrum, insula, supramarginal gyrus, SII, dIPFC, greater deactivation from verum in amygdala/ hippocampal formation. This heterogeneity suggests that groupderived contrast for verum and sham acupuncture tended to be above threshold in consistently specific brain areas, but were not significantly different in those areas, when assessed at the single study level.

\section{Strengths and limitations}

To our knowledge this is the first systematic and extensive review on $\mathrm{fMRI}$ and acupuncture without any language restrictions. Besides the internationally well known databases such as Pubmed and EMBASE, less well known international databases such as the Chinese CNKI, the Japanese Ichushi WEB, and the Korean NDSL and KTKP were searched and the publications found were included in this review. Therefore, this very extensive review provides a transparent and detailed overview of the current literature available. In addition we structured the publications according to the research questions, such as the differences in brain activity associated with acupuncture stimuli between patients and healthy volunteers, to provide a good overview and a strong basis for future study designs, interventions, measurement methods, and possible diagnoses. Moreover, we complemented the systematic and comprehensive literature review with several ALE meta-analyses, providing analytic results for stronger evidence that are supported statistically. However, some studies reported direct contrast between verum and sham acupuncture groups, while some others reported pre-post contrast for each group, resulting in the fact that several meta-analyses had to be performed. The studies included in the descriptive review and the meta-analyses were highly heterogeneous regarding their study design, their aims and their quality of reporting. The reasons for these heterogeneous results are numerous, such as the varying acupuncture manipulation methods, different types of control arms, different methods of acquisition and analyzing the imaging data, the mainly investigated brain regions (region of interest) and the statistical analysis. The large variability between subjects and sessions with respect to the imaging data also needs to be taken into consideration [39,154]. The imprecise nomenclature [155] is sometimes misleading, such as activation, deactivation, changes, baseline. We did not formally assess the quality of the publications, because no valid checklist for this type of research is available, though reporting guidelines are available and should be consulted by future research publications [156]. A narrative review including only studies that are considered to be of high quality would have overcome this problem. However the aim of this paper was to provide a systematic and broad overview for the first time using the publications currently available. We believe that many trials included in this review have limitations regarding their study design, analysis and reporting of their results. Hence, our results have to be interpreted with care. This is underlined by the multitude of contradictory results. Lastly, the field of research on brain imaging for acupuncture is evolving rapidly which may indeed lessen the relevance of older results using sub-optimal methodologies and analysis techniques. 
A. Brain response to verum acupuncture stimuli relative to rest
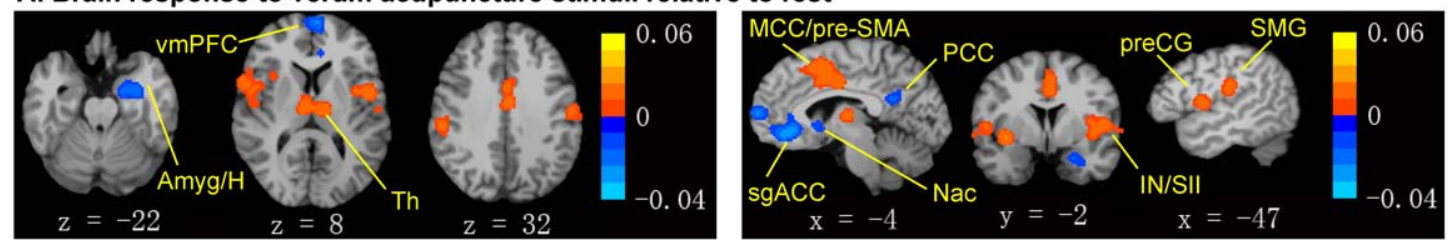

red: verum > rest $(1 \mathrm{a})$, blue: rest $>$ verum $(1 \mathrm{~b})$

B. Differences in brain response for verum and sham acupuncture from direct contrast

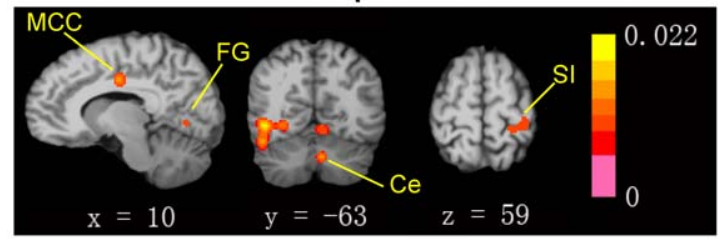

verum $>\operatorname{sham}(2 a)$

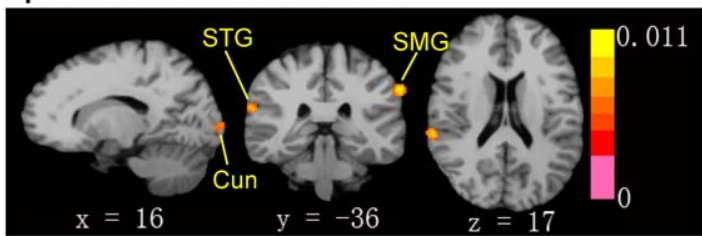

sham $>$ verum $(2 b)$

C. Brain response to verum and sham acupuncture stimuli relative to rest

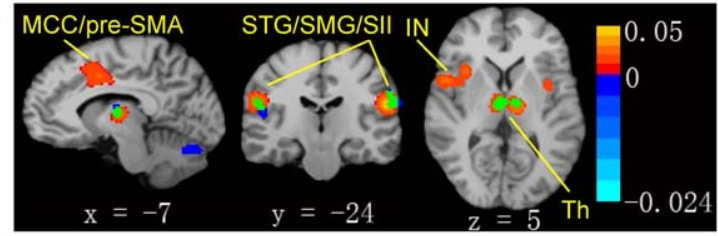

red: verum > rest $(3 a)$, blue: sham > rest $(3 c)$, green:overlap

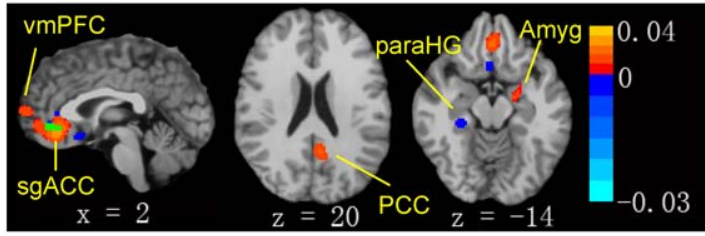

red: rest > verum (3b), blue: rest > sham (3d), green:overlap

D. Differences in brain response for verum and sham acupuncture from subtraction analysis

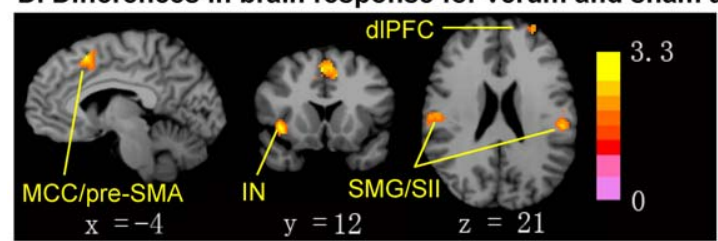

"verum > rest" - "sham > rest" (3e)

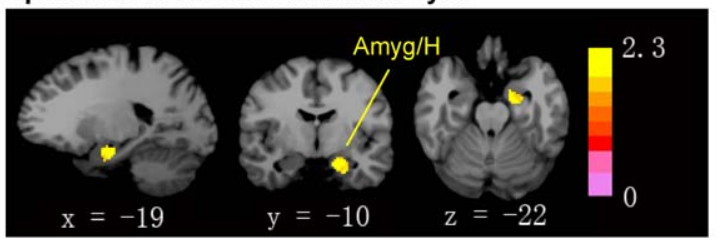

"rest > verum" - "rest > sham" (3f)

Figure 4. Results from the ALE meta-analyses. Meta-analyses were performed to evaluate brain response to acupuncture across studies, and contrast verum and sham acupuncture. (A) Brain response to verum acupuncture demonstrated activation in sensorimotor and affective/salience processing brain regions and deactivation in the amygdala and DMN brain regions. (B) Differences in brain response for verum and sham acupuncture from direct contrast showed significance in somatosensory areas, limbic regions, visual processing regions and cerebellum. (C) Brain response to verum and sham acupuncture individually demonstrated activation in sensorimotor and affective/salience processing brain regions and deactivation in the amygdala and DMN brain regions associated with verum acupuncture; while sham acupuncture produced activation in somatosensory regions, affective/salience processing regions, cerebellum and deactivation in limbic regions. (D) Differences in brain response between verum and sham acupuncture from subtraction analysis showed more activation in the sensorimotor affective/cognitive processing brain regions and more deactivation in the amygdala/hippocampal formation for verum acupuncture. For subfigures $A-C, p<0.05$, cluster level $F D R$ corrected, color bar showed ALE value; for subfigure $D, p<0.05$, cluster level uncorrected, color bar showed $Z$ value. Amyg: amygdala; Ce: cerebellum; dIPFC: dorsolateral prefrontal cortex; FG: fusiform gyrus; H: hippocampal formation; IN: insula; MCC: middle cingulate cortex; Nac: nucleus accumbens; paraHG: parahippocampal gyrus; PCC: posterior cingulate cortex; preCG: precentral gyrus; pre-SMA: pre-supplementary motor area; SI: primary somatosensory cortex; SII: secondary somatosensory cortex; SgACC: subgenual anterior cingulate cortex; SMG: supramarginal gyrus; Th: thalamus; vmPFC: ventromedial prefrontal cortex.

doi:10.1371/journal.pone.0032960.g004

\section{Discussion of results}

The studies on BOLD activation and deactivation from a single point or a group of points came mainly from China and Korea. The controlled studies, including sham acupuncture as a control, were mainly from China and the US: the Chinese studies mainly used penetrating sham at a nearby non-acupuncture point as a control while the US studies mainly applied the non-penetrating Streitberger needle or monofilament tapping at the same acupuncture points. Studies on patients were mainly from China. Although we did not evaluate the quality of the publications, the papers published in English used a clearer reporting style than those published in other languages. The most innovative studies came from the US. These studies had clear study questions and explored acupuncture neurocorrelates with a pain matrix, expectation, autonomic regulation, somatosensory perception and deqi related brain response.

While in the descriptive analysis similarities were observed in the brain response to stimulation at different acupuncture points, some differences across points were also noted. For example, brain deactivation observed in the visual areas (precuneus, cuneus) appeared not only when the vision related points (GB37, UB60) were needled, but also when several non-vision related points (LR2, LR3, ST36) were needled, but not with the other points. One could argue, based on TCM theory, that for the two points on liver meridian (LR2, LR3), the liver opens into the eyes, reflecting its physiological and pathological conditions [157]. The 
Table 5. Clusters showing significant convergence for verum versus sham acupuncture (FDR pN corrected at the cluster level, $p<0.05)$ from ALE meta-analyses.

\begin{tabular}{|c|c|c|c|c|c|c|c|}
\hline \multirow[t]{2}{*}{ Brain region } & \multirow[t]{2}{*}{ BA } & \multicolumn{3}{|c|}{ Talairach coordinates } & \multirow[t]{2}{*}{ ALE value } & \multirow[t]{2}{*}{$Z$ value } & \multirow[t]{2}{*}{ Volume $\left(\mathrm{mm}^{3}\right)$} \\
\hline & & $\mathbf{x}$ & $\mathbf{y}$ & $\mathbf{z}$ & & & \\
\hline \multicolumn{8}{|l|}{ Direct comparision verum >sham (2a) } \\
\hline Fusiform gyrus & 37 & 44 & -64 & -6 & 0.0197 & & 3720 \\
\hline Culmen of Vermis & & -2 & -66 & -10 & 0.0160 & & 1520 \\
\hline Cerebellar tonsil & & -4 & -58 & -32 & 0.0224 & & 1240 \\
\hline Postcentral gyrus & 3 & -20 & -36 & 64 & 0.0116 & & 992 \\
\hline Middle cingulate & 24 & 10 & -12 & 34 & 0.0165 & & 904 \\
\hline \multicolumn{8}{|l|}{ Direct comparision sham $>$ verum $(2 b)$} \\
\hline Supramarginal gyrus & 40 & -62 & -34 & 34 & 0.0108 & & 1120 \\
\hline Superior temporal gyrus & 42 & 64 & -34 & 20 & 0.0088 & & 552 \\
\hline Cuneus & 18 & 18 & -98 & 0 & 0.0069 & & 400 \\
\hline \multicolumn{8}{|l|}{ Verum>rest (3a) } \\
\hline Middle cingulate/Pre-supplementary motor area & 24 & -2 & 2 & 38 & 0.0353 & & 7392 \\
\hline Superior temporal gyrus & 22 & 50 & 6 & 2 & 0.0262 & & 6232 \\
\hline \multirow[t]{2}{*}{ Supramarginal gyrus/SII } & 40 & -54 & -22 & 18 & 0.0484 & & 6040 \\
\hline & 40 & 56 & -26 & 22 & 0.0402 & & 4080 \\
\hline Thalamus & & -8 & -16 & 6 & 0.0326 & & 4504 \\
\hline Insula & 13 & -38 & -4 & 0 & 0.0177 & & 2056 \\
\hline \multicolumn{8}{|l|}{ Sham >rest (3c) } \\
\hline Tuber of vermis & & 0 & -70 & -24 & 0.0239 & & 2664 \\
\hline Supramarginal gyrus/SII & 40 & -60 & -22 & 22 & 0.0159 & & 2424 \\
\hline \multirow[t]{2}{*}{ Superior temporal gyrus } & 41 & 50 & -32 & 16 & 0.0168 & & 2320 \\
\hline & 22 & -52 & 10 & -2 & 0.0180 & & 808 \\
\hline Thalamus & & 6 & -14 & 8 & 0.0206 & & 1848 \\
\hline \multicolumn{8}{|l|}{ Rest>verum (3b) } \\
\hline Anterior cingulate & 32 & 0 & 32 & -8 & 0.0413 & & 5400 \\
\hline Amygdala/hippocampal formation & 34 & -18 & -8 & -20 & 0.0262 & & 2632 \\
\hline Ventromedial prefrontal cortex & 10 & -2 & 60 & 10 & 0.0260 & & 1784 \\
\hline Posterior cingulate & 31 & -6 & -56 & 22 & 0.0193 & & 1288 \\
\hline \multicolumn{8}{|l|}{ Rest>sham (3d) } \\
\hline Anterior cingulate & 32 & -4 & 40 & -2 & 0.0128 & & 1720 \\
\hline Parahippocampal gyrus & 36 & 28 & -32 & -14 & 0.0110 & & 392 \\
\hline Subcallosal gyrus & 25 & 4 & 14 & -12 & 0.0108 & & 360 \\
\hline \multicolumn{8}{|l|}{ “Verum>rest" - "sham>rest" (3e)* } \\
\hline Pre-supplementary motor area/middle cingulate & 6 & 4 & 12 & 46 & & 2.7822 & 2120 \\
\hline Claustrum/insula & & 32 & 5 & -1 & & 3.2905 & 1848 \\
\hline \multirow[t]{2}{*}{ Supramarginal gyrus/SII } & 40 & -52 & -26 & 22 & & 2.4181 & 1728 \\
\hline & 40 & 54 & -18 & 24 & & 2.2904 & 1168 \\
\hline Dorsolateral prefontral cortex & 10 & -28 & 57 & 23 & & 2.2383 & 568 \\
\hline \multicolumn{8}{|l|}{ “Rest>verum" - "rest>sham” (3f)* } \\
\hline Amygdala/hippocampal formation & 34 & -14 & -9 & -20 & & 2.2768 & 1104 \\
\hline
\end{tabular}

stimulation of different acupoints in the same spinal segment could induce different fMRI activation patterns in the brain [142] while acupoints on the same meridian show some similarities in the activation/deactivation pattern [23].

The meta-analyses could only be done for publications that provided Talairach data, which was not the case for all of our study questions. The meta-analyses on the specific effect of acupuncture that compared verum and sham acupuncture came up with heterogeneous results. The subtraction analyses reflected descriptive results more than the direct contrast analyses. For example, subtraction meta-analyses confirmed more activation from verum in basal ganglia and insula, more deactivation in the 
limbic region of amygdala/hippocampal formation associated with verum, while meta-analyses of direct contrast for verum and sham confirmed more activation in cerebellum associated with verum. The convergence of brain regions shown for these meta-analyses comparing verum and sham acupuncture overlapped for middle cingulate gyrus. The first reason for the heterogeneous results might be the literature heterogeneity. Only two publications had both pre-post and between-group comparison results [15,37]. Also, the different methods of acupuncture stimuli may have a strong impact of the result. Moreover, the direct contrast "verum $>$ sham" included either more activation from verum or more deactivation from the sham. Thus, the results of direct contrast "verum $>$ sham" and subtraction analysis "verum $>$ rest" "sham>rest" are not directly comparable. The ALE subtraction analysis for the comparison of verum versus sham acupuncture should be interpreted with caution because the groups are disparate in total number of foci. However, we refrained from randomly extracting experiments from the larger foci set [10], as this might have biased our results substantially. In particular, for the "rest>verum" - "rest>sham", extracting 5 experiments out of 15 from "rest>verum" could most probably influence the result by chance. The meta-analysis of direct contrast for "sham> verum" included only three experiments and 27 foci. Hence this analysis might be with not enough power and doesn't represent the general. Nevertheless, we could see that brain regions such as SII, insula, cingulate gyrus, amygdala/hippocampal formation and prefrontal cortices might be important when differentiating the acupuncture specific effect from sham acupuncture. Acupuncture analgesia is considered as one of the most important indications for clinical acupuncture treatment [158], and those brain regions mentioned above are associated with the pain neuromatrix and might contribute in explaining the mechanism of acupuncture specific analgesia.

\section{Comparisons with other reviews}

Some of the previous reviews [7,8,159-161] focused on a broader topic of neuroimaging techniques including EEG, PET, SPECT or MEG. Those reviews summarized research questions underlying certain acupuncture mechanisms, such as acupuncture analgesia, acupuncture placebo effect, specificity of meridian and acupuncture points, and acupuncture modulation on brain networks. They displayed the evidence for each research question and cited the relevant literature accordingly. However, in most cases the literature search was not transparently displayed. The other reviews [162-168] focusing on acupuncture and fMRI, had other emphases: Beissner et al. [162] focused on methodological problems, Cho et al. [163] explored neural substrates for hypothalamus-pituitary-adrenal axis and Chae et al. [164] reviewed traditional Korean acupuncture. The four Chinese narrative reviews on fMRI and acupuncture [165-168] discussed several research questions on the specific effects of acupuncture, such as different acupuncture points, manipulation methods, deqi or not deqi, and sham acupuncture. Our systematic literature review aimed to display the available studies as broad as possible and should offer a better and deeper overview on this topic, thus supporting future studies.

\section{References}

1. Liang F (2006) Acupuncture and Moxibustion. Shanghai: Shanghai Scientific and Technical Publishers.

2. Backer M, Hammes M, Sander D, Funke D, Deppe M, et al. (2004) Changes of cerebrovascular response to visual stimulation in migraineurs after repetitive sessions of somatosensory stimulation (acupuncture): a pilot study. Headache 44: 95-101.
Methodological consideration regarding future studies

One of the advantages for fMRI is that there are multiple possibilities by which experiments can be designed and data analyzed, providing information on different aspects of brain physiology. However, the inherent heterogeneity can complicate subsequent reviews and meta-analyses. Certain basic guidelines on proper statistical analyses of fMRI data should be followed, such as calculating difference maps if two conditions, such as brain response to stimulation at different acupoints, are to be contrasted. Furthermore, as suggested by Poldrack et al., publications relating to fMRI investigations of acupuncture should report all pertinent information relating to both imaging and acupuncture procedures [156]. Important topics include design and task specification, planned group comparisons, behavioral performance metrics, imaging details, data pre-processing, intersubject registration, statistical modeling details for both the individual and group level, and statistical inference including approach to multiple comparisons correction. Adoption of these guidelines will improve manuscript reviews and shorten the time to acceptance (or rejection), as well as facilitate the inclusion of publications in future reviews and meta-analyses.

\section{Conclusion}

Brain response to acupuncture stimuli encompasses a broad network of regions consistent with not just somatosensory, but also affective and cognitive processing. While published results on acupuncture and fMRI were heterogeneous, from a descriptive perspective most studies suggest that acupuncture can modulate the brain activity within specific brain areas, and the evidence based on meta-analyses confirmed part of these results. Future studies should further improve methodological aspects and reporting related to both fMRI and acupuncture, and strictly control experimental conditions for more robust inference. Specifically, direct contrast analyses should be used to contrast different stimulus conditions (e.g. verum versus sham acupuncture) when evaluating research questions concerning acupuncture specificity.

\section{Supporting Information}

Table S1 Descriptive analysis of differences between verum and sham acupuncture. (DOCX)

Table S2 Descriptive analysis of changes related to cortical and sub-cortical activation and deactivation at verum acupuncture points.

(DOCX)

\section{Acknowledgments}

We would like to thank the authors who provided us further details about their trial for our meta-analyses.

\section{Author Contributions}

Conceived and designed the experiments: WJH CW DP. Analyzed the data: WJH DP VN KP XYL JN YM CW. Wrote the paper: WJH DP VN KP XYL JN YM TN FRL CW. Data extraction: WJH DP KP YM.

3. WHO (2002) Acupuncture: Review and Analysis of Reports on Controlled Clinical Trials: World Health Organization. . 87 p.

4. Linde KSA, Jürgens S, Hoppe A, Brinkhaus B, Witt C, et al. (2005) Acupuncture for Patients with Migraine - A Randomized Trial (ART Migraine). JAMA 293: 2118-2125. 
5. Brinkhaus BWC, Jena S, Linde K, Streng A, Wagenpfeil S, et al. (2006) Acupuncture in Patients with Chronic Low Back Pain - A Randomised Controlled Trial. Arch Intern Med 166: 450-457.

6. Melchart D, Streng A, Hoppe A, Brinkhaus B, Witt C, et al. (2005) Acupuncture in patients with tension-type headache: randomised controlled trial. BMJ 331: 376-382.

7. Dhond RP, Kettner N, Napadow V (2007) Neuroimaging acupuncture effects in the human brain. J Altern Complement Med 13: 603-616.

8. Lewith GT, White PJ, Pariente J (2005) Investigating acupuncture using brain imaging techniques: the current state of play. Evid Based Complement Alternat Med 2: 315-319.

9. Eickhoff SB, Laird AR, Grefkes C, Wang LE, Zilles K, et al. (2009) Coordinate-based activation likelihood estimation meta-analysis of neuroimaging data: a random-effects approach based on empirical estimates of spatial uncertainty. Hum Brain Mapp 30: 2907-2926.

10. Laird AR, Fox PM, Price CJ, Glahn DG, Uecker AM, et al. (2005) ALE metaanalysis: controlling the false discovery rate and performing statistical contrasts. Hum Brain Mapp 25: 155-164.

11. Turkeltaub PE, Eden GF, Jones KM, Zeffiro TA (2002) Meta-analysis of the functional neuroanatomy of single-word reading: method and validation. Neuroimage 16: 765-780.

12. Lancaster JL, Tordesillas-Gutierrez D, Martinez M, Salinas F, Evans A, et al. (2007) Bias between MNI and Talairach coordinates analyzed using the ICBM-152 brain template. Hum Brain Mapp 28: 1194-1205.

13. Eickhoff SB, Bzdok D, Laird AR, Roski C, Caspers S, et al. (2011) Coactivation patterns distinguish cortical modules, their connectivity and functional differentiation. Neuroimage 57: 938-949.

14. Streitberger K, Kleinhenz J (1998) Introducing a placebo needle into acupuncture research. Lancet 352: 364-365.

15. Yoo SS, Teh EK, Blinder RA, Jolesz FA (2004) Modulation of cerebellar activities by acupuncture stimulation: evidence from fMRI study. Neuroimage 22: 932-940

16. Yoo SS, Kerr CE, Park M, Im DM, Blinder RA, et al. (2007) Neural activities in human somatosensory cortical areas evoked by acupuncture stimulation. Complement Ther Med 15: 247-254.

17. Li G, Jack CR, Jr., Yang ES (2006) An fMRI study of somatosensoryimplicated acupuncture points in stable somatosensory stroke patients. J Magn Reson Imaging 24: 1018-1024.

18. Chae Y, Lee H, Kim H, Kim CH, Chang DI, et al. (2009) Parsing brain activity associated with acupuncture treatment in Parkinson's diseases. Mov Disord 24: 1794-1802.

19. Schaechter JD, Connell BD, Stason WB, Kaptchuk TJ, Krebs DE, et al. (2007) Correlated change in upper limb function and motor cortex activation after verum and sham acupuncture in patients with chronic stroke. J Altern Complement Med 13: 527-532.

20. Schockert T, Schnitker R, Boroojerdi B, Vietzke K, Qua Smith I, et al. (2009) Kortikale Aktivierungen durch Yamamoto Neue Schädelakupunktur (YNSA) in der Behandlung von Schlaganfallpatienten: Eine Sham-kontrollierte Studie mit Hilfe der funktionellen Kernspintomographie (fMRI). Deutsche Zeitschrift für Akupunktur 52: 21-29.

21. Dougherty DD, Kong J, Webb M, Bonab AA, Fischman AJ, et al. (2008) A combined [11C]diprenorphine PET study and fMRI study of acupuncture analgesia. Behav Brain Res 193: 63-68.

22. Deng G, Hou BL, Holodny AI, Cassileth BR (2008) Functional magnetic resonance imaging (fMRI) changes and saliva production associated with acupuncture at LI-2 acupuncture point: a randomized controlled study. BMC Complement Altern Med 8: 37.

23. Li L, Liu H, Li YZ, Xu JY, Shan BC, et al. (2008) The human brain response to acupuncture on same-meridian acupoints: evidence from an fMRI study. J Altern Complement Med 14: 673-678.

24. Xiao YY, Du L, Hong BK, et al. (2008) Study on fMRI brain map in patients undergoing needling at Zusanli (ST36) by reinforcing method. Zhongguo Zhong Xi Yi Jie He Za Zhi 28: 122-125.

25. Zhang JH, Cao XD, Lie J, Tang WJ, Liu HQ, et al. (2007) Neuronal specificity of needling acupoints at same meridian: a control functional magnetic resonance imaging study with electroacupuncture. Acupunct Electrother Res 32: $179-193$.

26. Hu KM, Wang CP, Xie HJ, Henning J (2006) Observation on activating effectiveness of acupuncture at acupoints and non-acupoints on different brain regions. Zhongguo Zhen Jiu 26: 205-207.

27. Wang W, Li KC, Shan BC, Xu JY, Yan B, et al. (2006) Study of acupuncture point Liv 3 with funcitonal MRI. Chin J Radiol 40: 29-35.

28. Yan B, Li K, Xu J, Wang W, Liu H, et al. (2005) Acupoint-specific fMRI patterns in human brain. Neurosci Lett 383: 236-240.

29. Li G, Liu HL, Cheung RT, Hung YC, Wong KK, et al. (2003) An fMRI study comparing brain activation between word generation and electrical stimulation of language-implicated acupoints. Hum Brain Mapp 18: 233-238.

30. Lu N, Zhao JG, Shan BC, Li KC (2008) Study of acupuncture point Liv6 with functional MRI. Chin J Med Imaging Technol 24: 46-48.

31. Huang Y, Li GL, Lai XS, Tang CZ, Yang JJ (2009) An fMRI cerebral functional imaging comparison on needling in Zhigou (SJ6) vs a sham point. Journal of Chengdu University of TCM 32: 3-6.
32. Li L, Xu JY, Li YZ, Liu H, Yuan XL, et al. (2008) fMRI study of acupuncture at adjacent acupoints located on the stomach meridian of Foot-Yanming. Chin J Med Imaging Technol 24: 1001-1003.

33. Lai XS, Su PZ, Huang Y, Zou YQ, Wu JX, et al. (2009) Comparison of fMRI cerebral functional imaging between needling Waiguan (SJ5) and combined SJ5 and sham point. Tianjin Journal of Traditional Chinese Medicine 26: 113-115.

34. Jeun SS, Kim JS, Kim BS, Park SD, Lim EC, et al. (2005) Acupuncture stimulation for motor cortex activities: a 3T fMRI study. Am J Chin Med 33: 573-578.

35. Choi N-g, Han J-b, Jang S-j (2009) Comparaison of brain activation iamges associated with sexual arousal induced by visual stimulation and SP6 acupucnture : fMRI at 3 tesla. JOURNAL OF RADIOLOGICAL SCIENCE AND TECHNOLOGY 32: 183-194.

36. Choe B-y (2002) Clinical Application of Functional MRI : Motor Cortex Activities by Acupuncture. Journal of the Korean Magnetic Resonance Society 6: 89-93.

37. Wu MT, Sheen JM, Chuang KH, Yang P, Chin SL, et al. (2002) Neuronal specificity of acupuncture response: a fMRI study with electroacupuncture. Neuroimage 16: 1028-1037.

38. Zhang JH, Feng XY, Li J, Tang WJ, Li Y (2005) Functional MRI studies of acupoints and non-acupoints electroacupuncture analgesia modulating within human brain. Chinese computed medical imaging 11: 10-16.

39. Kong J, Gollub RL, Webb JM, Kong JT, Vangel MG, et al. (2007) Test-retest study of fMRI signal change evoked by electroacupuncture stimulation. Neuroimage 34: 1171-1181.

40. Kong J, Kaptchuk TJ, Webb JM, Kong JT, Sasaki Y, et al. (2007) Functional neuroanatomical investigation of vision-related acupuncture point specificity-A multisession fMRI study. Hum Brain Mapp.

41. Cho Z-H, Hwang S-c, Son Y-d, Kang C-k, Wong EK, et al. (2004) Acupuncture Analgesia : A Sensory Stimulus Induced Analgesia Observed by functional Magnetic resonance Imaging. The Journal of Korean Acupuncture \& Moxibustion Society 21: 57-71.

42. Fang JL, Krings T, Weidemann J, Meister IG, Thron A (2004) Functional MRI in healthy subjects during acupuncture: different effects of needle rotation in real and false acupoints. Neuroradiology 46: 359-362.

43. Wesolowski T, Lotze M, Domin M, Langner S, Lehmann C, et al. (2009) Acupuncture reveals no specific effect on primary auditory cortex: a functional magnetic resonance imaging study. Neuroreport 20: 116-120.

44. Wang GB, Liu C, Wu LB, Yan B, Gao SZ, et al. (2009) Functional magnetic resonance imaging on acupuncturing Yuan-Source and He-Sea acupoints of stomach meridian of foot Yangming. ACTA ACADEMIAE MEDICINAE SINICAE 31: 171-176.

45. Fang JL, Jin Z, Wang Y, Li K, Zeng YW, et al. (2005) Comparison of central effects of acupuncturing Taichong and nearby two acupoints by functional MRI. Chin J Med Imaging Technol 21: 1332-1336.

46. FangJ, Jin Z, Wang Y, Li K, KongJ, et al. (2008) The salient characteristics of the central effects of acupuncture needling: Limbic-paralimbic-neocortical network modulation. Hum Brain Mapp.

47. Wang W, Liu L, Zhi X, Huang JB, Liu DX, et al. (2007) Study on the regulatory effect of electro-acupuncture on hegu point (LI4) in cerebral response with functional magnetic resonance imaging. Chin J Integr Med 13: $10-16$

48. Ai L, Dai JP, Zhao BX, Tian J, Fan YP, et al. (2004) Investigation of analgesic mechanism of acupuncture : a fMRI study. Chin J Med Imaging Technol 20: 1197-1200.

49. Li G, Huang L, Cheung RT, Liu SR, Ma QY, et al. (2004) Cortical activations upon stimulation of the sensorimotor-implicated acupoints. Magn Reson Imaging 22: 639-644.

50. Liu WC, Feldman SC, Cook DB, Hung DL, Xu T, et al. (2004) fMRI study of acupuncture-induced periaqueductal gray activity in humans. Neuroreport 15: 1937-1940.

51. Napadow V, Dhond R, Park K, Kim J, Makris N, et al. (2009) Time-variant fMRI activity in the brainstem and higher structures in response to acupuncture. Neuroimage 47: 289-301.

52. Wu MT, Hsieh JC, Xiong J, Yang CF, Pan HB, et al. (1999) Central nervous pathway for acupuncture stimulation: localization of processing with functional MR imaging of the brain-preliminary experience. Radiology 212: 133-141.

53. Hui KK, Liu J, Marina O, Napadow V, Haselgrove C, et al. (2005) The integrated response of the human cerebro-cerebellar and limbic systems to acupuncture stimulation at ST 36 as evidenced by fMRI. Neuroimage 27: 479-496.

54. Hui KK, Marina O, Claunch JD, Nixon EE, Fang J, et al. (2009) Acupuncture mobilizes the brain's default mode and its anti-correlated network in healthy subjects. Brain Res 1287: 84-103.

55. Napadow V, Dhond RP, Kim J, LaCount L, Vangel M, et al. (2009) Brain encoding of acupuncture sensation-coupling on-line rating with fMRI. Neuroimage 47: 1055-1065.

56. Fukunaga M (1999) Brain activation under electro-acupuncture stimulation using functional magnetic resonance imaging. The bulletin of Meiji University of oriental medicine 25: 7-19.

57. Dhond RP, Yeh C, Park K, Kettner N, Napadow V (2008) Acupuncture modulates resting state connectivity in default and sensorimotor brain networks. Pain 136: 407-418. 
58. Napadow V, Makris N, Liu J, Kettner NW, Kwong KK, et al. (2005) Effects of electroacupuncture versus manual acupuncture on the human brain as measured by fMRI. Hum Brain Mapp 24: 193-205.

59. Hui KK, Liu J, Makris N, Gollub RL, Chen AJ, et al. (2000) Acupuncture modulates the limbic system and subcortical gray structures of the human brain: evidence from fMRI studies in normal subjects. Hum Brain Mapp 9: $13-25$

60. Chae Y, Lee H, Kim H, Sohn H, Park JH, et al. (2009) The neural substrates of verum acupuncture compared to non-penetrating placebo needle: an fMRI study. Neurosci Lett 450: 80-84.

61. Huang Y, Zeng TJ, Wang YJ, Lai XS, Zhang YZ, et al. (2009) A comparison of functional magnetic resonance imaging of cerebral regions activated by cutaneous needling and regular needling at Waiguan (SJ5) acupoint. Journal of Anhui TCM college 28: 25-28.

62. Wu JX, Huang Y, Lai XS, Zou YQ Tang CZ, et al. (2009) The fMRI comparative study on cutaneous or routine needling in acupoints Waiguan (SJ5) and Neiguan (PC6). Chinese archives of traditional Chinese medicine 27: $1625-1627$

63. Huang Y, Song YB, Lai XS, Tang CZ, Yang JJ (2009) Comparison study between shallowly skin needling and routine needling in Neiguan (PC6) by fMRI. Journal of Shandong University of TCM 33: 243-245.

64. Huang Y, LI XX, Lai XS, Zou YQ, Wu JX (2009) Study of fMRI of cerebral functional regions induced by cutaneous and routine needling in Zhigou (SJ6). Hebei J TCM 31: 254-256.

65. Zou YQ, Huang Y, Lai XS, Tang CZ, Yang JJ (2008) The fMRI comparative study on cutaneous or routine needling in acupoints Waiguan (SJ5) and Zhigou (SJ6). Journal of Yunnan University of traditional Chinese medicine 31: $44-47$.

66. Li G, Cheung RT, Ma QY, Yang ES (2003) Visual cortical activations on fMRI upon stimulation of the vision-implicated acupoints. Neuroreport 14: 669-673.

67. MacPherson H, Green G, Nevado A, Lythgoe MF, Lewith G, et al. (2008) Brain imaging of acupuncture: comparing superficial with deep needling. Neurosci Lett 434: 144-149.

68. Li DZ, Li XZ (2000) Comparative study of pricking the cutaneous region of acupoint and needling its depth by means of fMRI. Zhongguo Zhen Jiu. pp $491-492$

69. Kong J, Ma L, Gollub RL, Wei J, Yang X, et al. (2002) A pilot study of functional magnetic resonance imaging of the brain during manual and electroacupuncture stimulation of acupuncture point (LI-4 Hegu) in normal subjects reveals differential brain activation between methods. J Altern Complement Med 8: 411-419.

70. Li K, Shan B, Xu J, Liu H, Wang W, et al. (2006) Changes in FMRI in the human brain related to different durations of manual acupuncture needling. J Altern Complement Med 12: 615-623.

71. Hu KM, Wang CP, Henning J (2005) Observation on relation of acupuncture at Guangming (GB 37) and Taichong (LR 3) with central nervous reaction. Zhongguo Zhen Jiu 25: 860-862.

72. Gareus IK, Lacour M, Schulte AC, Hennig J (2002) Is there a BOLD response of the visual cortex on stimulation of the vision-related acupoint GB 37? J Magn Reson Imaging 15: 227-232.

73. Cheng HJ, Chen SJ, Zhu F (2009) Magnetic resonance imaging study of twisting or untwisting Taixi acupoint (KI3) on brain function. Journal of clinical rehabilitative tissue engineering research 13: 5020-5022.

74. Gong HH, Wang YZ, Xiao XZ, Qiu CM, Wang LY, et al. (2003) Investigation of cerebral cortical functional areas of the acupoints in zu-san-li and xia-ju-xu by fMRI. Journal of diagnostic imaging \& interventional radiology 12: $133-136$

75. Wang W, Qi JP, Xia YL, Huang XL, Li WX, et al. (2004) The response of human motor cortex to acupuncture of S36 and G34 as revealed by functional MRI. Chin J Phys Med Rehabil 26: 472-475.

76. Fu P, Jia JP, Zhu J, Huang.JJ (2005) [Effects of acupuncture at Neiguan (PC 6) on human brain functional imaging in different functional states]. Zhongguo Zhen Jiu 25: 784-786.

77. Liu S, Zhou W, Ruan X, Li R, Lee T, et al. (2007) Activation of the hypothalamus characterizes the response to acupuncture stimulation in heroin addicts. Neurosci Lett 421: 203-208.

78. Wu Y, Jin Z, Li K, Lu ZL, Wong V, et al. (2008) Effect of acupuncture on the brain in children with spastic cerebral palsy using functional neuroimaging (FMRI). J Child Neurol 23: 1267-1274.

79. Napadow V, Liu J, Li M, Kettner N, Ryan A, et al. (2007) Somatosensory cortical plasticity in carpal tunnel syndrome treated by acupuncture. Hum Brain Mapp 28: 159-171.

80. Napadow V, Kettner N, Liu J, Li M, Kwong KK, et al. (2007) Hypothalamus and amygdala response to acupuncture stimuli in Carpal Tunnel Syndrome. Pain 130: 254-266.

81. Hu JP, Li YG, Cao DR, Gong SB (2008) Reproducibility of functional MR imaging during electroacupuncture stimulation at PC6 (Neiguan). ACTA ACADEMIAE MEDICINAE MILITARIS TERTIAE 30: 1878-1882.

82. Fu P, Jia JP, Min BQ (2005) Acupuncture at Neiguan acupoint for brain functional MRI of patients with Alzheimer disease. Chin J Neurol. pp $118-119$.

83. Ha C-h, Lee H, Lim Y-k, Hong K-e, Lee B-r, et al. (2003) A fMRI study on the cerebral activity induced by Electro-acupuncture on Taichong(Liv3). The Journal of Korean Acupuncture \& Moxibustion Society 20: 187-207.
84. Xu FM, Xie P, Lv FJ, Mou J, Li YM, et al. (2007) The fMRI study of acupuncture at the five transport points of liver meridian. Journal of Nanjing TCM University 23: 224-227.

85. Yan LP, Sun ZR, Xie B, Ma X (2005) Brain functional response after electroacupuncture at Quchi point with fMRI method. JCAM 21: 61-63.

86. Hong K-e, Lee B-r, Lee H, Yim Y-k, Kim Y-j (2003) A fMRI study on the cerebral activity induced by Electro-acupuncture on Sanyinjiao(Sp6). The Journal of Korean Acupuncture \& Moxibustion Society 20: 86-103.

87. Parrish TB, Schaeffer A, Catanese M, Rogel MJ (2005) Functional magnetic resonance imaging of real and sham acupuncture. Noninvasively measuring cortical activation from acupuncture. IEEE Eng Med Biol Mag 24: 35-40.

88. Kim J-h, Lee H, Lim Y-k, Hong K-e, Lee B-r, et al. (2003) A fMRI study on the cerebral activity induced by Electro-acupuncture on Sp9(Yinlingquan). The Journal of Korean Acupuncture \& Moxibustion Society 20: 114-133.

89. Zhu MJ, Hu KM (2004) A fMRI study of the TCM theory "the liver connects eye links". Journal of Hainan medical college 10: 169-170.

90. Bae E-j, Hong K-e, Lee H, Lee B-r, Yim Y-k, et al. (2003) A fMRI study on the cerebral activity induced by Electro-acupuncture on Fenglon(St40). The Journal of Korean Acupuncture \& Moxibustion Society 20: 208-226.

91. Chi X (2009) A fMRI study of acupuncture on Zhongzhu (SJ3) point. Acta Chinese medicine and pharmacology 37: 37-38.

92. Chi X, Ju YL, Sun ST (2007) Study of acupuncture Zhongzhu (SJ3) Houxi (SI3) by functional MRI (fMRI). Chinese archives of traditional Chinese medicine 25: 843-844.

93. Huang Y, Li TL, Lai XS, Zou YQ, Wu JX, et al. (2009) Functional brain magnetic resonance imaging in healthy people receiving acupuncture at Waiguan versus Waiguan plus Yanglingquan points: a randomized controlled trail. Journal of Chinese integrative medicine 7: 527-531.

94. Zhang R, Zou YQ Huang SQ Chen ZG, Liang BL, et al. (2007) MRI cerebral function imaging following acupuncture at Hegu, Zusanli, Neiguan and Sanyinjiao points. Journal of clinical rehabilitative tissue engineering research 11: 4271-4274.

95. Hou JW, Huang WH, Wang Q, Feng JW, Pu YL, et al. (2002) Functional MRI studies of acupuncture analgesia modulating within the human brain. Chin J Radiol 36: 206-210.

96. Wang W, Xu HB, Kong XQ, Huang JB, Zhi X, et al. (2009) Experimental study on fMRI in human brain with electroacupuncture. J Pract Radiol 25: 305-308.

97. Li K, Shan BC, Liu H, Wang W, Xu JY, et al. (2005) fMRI study of acupuncture at large intestine 4. Chin J Med Imaging Technol 21: 1329-1331.

98. Liu H, Shan BC, Gao DS, Xu JY, Wang W, et al. (2006) Different cerebellar responding to acupuncture at Liv3 and LI4: an fMRI study. Chin J Med Imaging Technol 22: 1165-1167.

99. Yin L, Jin XL, Shi X, TIan JH, Ma L, et al. (2002) Imaging with PET and fMRI on brain function in acupuncturing the ST36 (Zusanli). Chin J Rehabil Theory Practice 8: 523-524.

100. Xu JY, Wang FQ, Wang H, Shan BC, Lv J, et al. (2004) Control study on effects of acupuncture at Hegu (LI4) and Taichong (LR3) points on fMRI cerebral function imaging. Zhongguo Zhen Jiu 24: 263-265.

101. Long Y, Liu B, Liu X, Yan CG, Chen ZG, et al. (2009) Resting-state functional MRI evaluation of after-effect of acupuncture at Zusanli point. Chin J Med Imaging Technol 25: 373-376.

102. Xiao YY, Wu RH, Pei RQ, Lin R, Rao HB (2004) Functional MR imaging (fMRI) of acupuncture: observation of stimulating the acu-point ST36 (zusanli). J Pract Radiol 20: 106-108.

103. Fu P, Jia JP, Xu M, Wang M (2005) Changes of brain function in different areas of cerebral cotices due to electroacupuncture at the point ST36 through MRI. Chinese journal of clinical rehabilitation 9: 92-93.

104. Wu ZY, Miao F, Xiang QY, Hao J, Cao Y, et al. (2007) The MRI research of brain function on acupuncture at Zusanli (ST36) point. Chinese journal of traditional medical science and technology 14: 305-307.

105. Fang SH, Zhang SZ, Liu H (2006) Study on brain response to acupuncture by functional magnetic resonance imaging-observation on 14 healthy subjects. Zhongguo Zhong Xi Yi Jie He Za Zhi 26: 965-968.

106. Tian LF, Zhou C, Chen M, Zhou TG, Cai K, et al. (2006) Using functional magnetic resonance imaging to study the correlation between the acupoint and cerebral region. Zhen Ci Yan Jiu 31: 113-115.

107. Kim Y-i, Kim Y-h, Lim Y-k, Lee H, Lee B-r, et al. (2003) A fMRI study on the cerebral activity induced by Electro-acupuncture on Zusanli(St36). The Journal of Korean Acupuncture \& Moxibustion Society 20: 133-150.

108. Chi X, Sun ST (2007) Cerebral functional magnetic resonance imaging study of point Waiguan acupuncture. Shanghai J Acu-mox 26: 30-31.

109. Park T-g, Kim Y-I, Hong K-e, Yim Y-k, Lee H, et al. (2004) A study on Brain activity induced by electro-acupuncture on Taechung(LR3) and Hapkok(LI4) using functional Magnetic Resonance Imaging. The Korean Journal of Meridian \& Acupoint 21: 29-46.

110. Chen WJ, Shou YQ, LiJH, Xu ZS, Liu H (2007) The effect of acupuncture at the acupoint Sanyinjiao on brain function as revealed by the functional magnetic resonance imaging. Chin J Phys Med Rehabil 29: 774-779.

111. Wu ZY, Miao F, Xiang QY, Hao J, Ge LB, et al. (2008) Comparative study on acupuncturing the different acupoints of the same meridian with functional magnetic resonance imaging. Chinese J Med Imaging 16: 101-105.

112. Cai K, Chen M, Wang WC, Zhou C, Zhou TG, et al. (2007) fMRI of cortical activation by acupuncture. Information of medical equipment 22: 84-86. 
113. Guan YQ Yang XZ (2008) Brain BOLD-fMRI study of electroacupuncture stimulating the acupoint related visual. Hebei J TCM 30: 1065-1068.

114. Chen P, Zhao BX, Qin W, Chen HY, Tian J, et al. (2008) Study on the mechanism of acupuncture at Daling (PC 7) for mental diseases by fMRI. Zhongguo Zhen Jiu 28: 429-432.

115. Park K-y, Lee B-r, Lee H, Yim Y-k, Hong K-e, et al. (2003) A fMRI study on the cerebral activity induced by Electro-acupuncture on Taixi(K3). The Journal of Korean Acupuncture \& Moxibustion Society 20: 194-208.

116. Yoon J-h, Hwang M-s, Bae G-t, Lee S-h, Lee S-d, et al. (2001) The new finding on BOLD response of motor acupoint KI6 by fMRI. The Journal of Korean Acupuncture \& Moxibustion Society 18: 60-69.

117. Kwon C-h, Lee J-b, Hwang M-s, Yoon J-h (2004) The New Finding on BOLD Response of Moter Acupoint KI6 by fMRI. The Journal of Korean Acupuncture \& Moxibustion Society 21: 177-186.

118. Kang J-h, Lee H, Lee B-r, Hong K-e, Yim Y-k, et al. (2003) A fMRI study on the cerebral activity induced by Electro-acupuncture on K7(Fuliu). The Journal of Korean Acupuncture \& Moxibustion Society 20: 66-84.

119. Zhang Y, LiangJ, Qin W, Liu P, von Deneen KM, et al. (2009) Comparison of visual cortical activations induced by electro-acupuncture at vision and nonvision-related acupoints. Neurosci Lett 458: 6-10.

120. Chi X, Sun ST, Bao DP (2006) fMRI study of acupuncture at Houxi (SI3) point. JCAM 22: 37-38

121. Chen SJ, Liu B, Fu WB, Wu SS, Chen J, et al. (2008) A fMRI observation on different cererbral regions activated by acupuncture of Shenmen (HT 7) and Yanglao (SI 6). Zhen Ci Yan Jiu 33: 267-271.

122. Chen HD, Ying GL, Jiang B, He WL (2006) Studding effect of acupuncturing Baihui point for brain function with fMRI method. Journal of Zhejiang University of traditional Chinese medicine 30: 656-659.

123. Wang AC, Wang YL, Jiang T, Ma B, Chen JF, et al. (2005) Observation on changes of cerebral images after acupuncture of Shenmai (BL62) by using fMRI. Zhen Ci Yan Jiu 30: 43-47.

124. Deng ZS, Qiu ML, C. F-S SL (2005) A study on response of the specific functional areas of the human brain to acupuncture stimulating visual-ralated acupoints using BOLD fMRI. J Pract Radiol 21: 1240-1242.

125. Rheu K-h, Choi I-h, Park H-j, Lim S (2006) fMRI Study on the Brain Activity Induced by Manual Acupuncture at BL62. The Korean Journal of Meridian \& Acupoint 23: 89-103.

126. Fu P, Jia JP, Wang M (2005) Acupuncture at Shenmen acupoint for brain functional MRI of patients with Alzheimer disease. Chinese journal of clinical rehabilitation. pp 120-121.

127. Bai L, Qin W, Tian J, Liu P, Li L, et al. (2009) Time-varied characteristics of acupuncture effects in fMRI studies. Hum Brain Mapp 30: 3445-3460.

128. Wang W, Zhu F, Qi JP, Xia YL, Xia LM, et al. (2002) Comparison study of human brain response to acupuncture stimulation vs finger tapping task by using real time fMRI. Chin J Radiol 36: 211-214

129. Wang SM, Constable RT, Tokoglu FS, Weiss DA, Freyle D, et al. (2007) Acupuncture-induced blood oxygenation level-dependent signals in awake and anesthetized volunteers: a pilot study. Anesth Analg 105: 499-506.

130. Zhen JP, Liu C, He JZ, Wang BG, Yang DH, et al. (2008) The research of brain $\mathrm{fMRI}$ in acupuncture of $\mathrm{KI}$ in different time. Chinese imaging journal of integrated traditional and western medicine 6: 325-331.

131. Park J-m, Gwak J-y, Cho S-y, Park S-u, Jung W-a, et al. (2008) Effects of Head Acupuncture Versus Upper and Lower Lims Acupuncture on Signal Activation of Blood Oxygen Level Dependent (BOLD) fMRI on the Brain and Somatosensory Cortex. The Journal of Korean Acupuncture \& Moxibustion Society 25: 151-165.

132. Teng J, Wang YL, Wang AC, Zhao JN, Liu M (2008) Study on cerebral images with frontal-three-needle penetration by functional magnetic resonance imaging. Journal of Shandong University of TCM 32: 104-106.

133. Zhou Y, Jin J (2008) Effect of acupuncture given at the HT 7, ST 36, ST 40 and KI 3 acupoints on various parts of the brains of Alzheimer's disease patients. Acupunct Electrother Res 33: 9-17.

134. LiJ, ZhangJH, DongJC (2007) Influence of acupuncture analgesia on cerebral function imaging in sciatica patients. Shanghai J Acu-mox 26: 3-6.

135. Chang JL, Gao Y, Zhang H, Tan ZJ, Jiang GD (2007) A preliminary discussion of the effect of electroacupuncture at acupoints HT5 and GB39 on lingual function and fMRI changes in a case of subcortical aphasia. Chinese journal of rehabilitation medicine 22: 13-17.

136. Wang W, Li KC, Shan BC, Yan B, Hao J, et al. (2006) fMRI study of acupuncture at "four gate points" on normal aging people. Chin J Med Imaging Technol 22: 829-832.

137. Zhou C, Wang JZ, Chen M, Zhou TG, Cai K, et al. (2005) The correlative study between acupoint stimulations and corresponding brain cortices on functional MRI. Chin J Radiol 39: 252-255.

138. He YZ, Wang LN, Huang L, Wang XH, Liu SR, et al. (2006) Effects of acupuncture on the cortical functional areas activated by index finger motion in the patient with ischemic stroke. Zhongguo Zhen Jiu 26: 357-361.

139. Xu FM, Xie P, Lu FJ, Mou J, Li YM, et al. (2007) Study on corresponding areas the liver and lung channels in brain with fMRI. Zhongguo Zhen Jiu 27: $749-752$.
140. Qiu MG, Wang J, Xie B, Wu BH, Zhang SX, et al. (2005) Establishment of analyzing methods for functional MR images when electroacupuncture stimulation on Guangming and Waiguan acupoints. ACTA ACADEMIAE MEDICINAE MILITARIS TERTIAE 27: 1970-1972.

141. Xu JY, Wang FQ, Shan BC, Chen Y, Wang H, et al. (2004) PET and fMRI to evaluate the results of acupuncture treatment of the cognition of alzheimer's disease. Chinese imaging journal of integrated traditional and western medicine 2: 85-87.

142. Zhang WT, Jin Z, Luo F, Zhang L, Zeng YW, et al. (2004) Evidence from brain imaging with fMRI supporting functional specificity of acupoints in humans. Neurosci Lett 354: 50-53.

143. Cho Z-h, Kim K-y, Kim H-k, Lee B-r, Wong EK, et al. (2001) Correlation between acupuncture stimulation and cortical activation - further evidence. The Journal of Korean Acupuncture \& Moxibustion Society 18: 105-113.

144. Chang SX, Feng GS, Kong XQ Li G, Liu DX, et al. (2002) Functional MRI study of cortical function area activation with electronic acupuncture stimulation of multiple acupoints. Lin Chuang Fang She Xue Za Zhi 21: 99-102.

145. Kong J, Kaptchuk TJ, Polich G, Kirsch I, Vangel M, et al. (2009) An fMRI study on the interaction and dissociation between expectation of pain relief and acupuncture treatment. Neuroimage 47: 1066-1076.

146. Kong J, Kaptchuk TJ, Polich G, Kirsch I, Vangel M, et al. (2009) Expectancy and treatment interactions: a dissociation between acupuncture analgesia and expectancy evoked placebo analgesia. Neuroimage 45: 940-949.

147. Kong J, Gollub RL, Rosman IS, Webb JM, Vangel MG, et al. (2006) Brain activity associated with expectancy-enhanced placebo analgesia as measured by functional magnetic resonance imaging. J Neurosci 26: 381-388.

148. Zhang Y, Oin W, Liu P, Tian J, Liang J, et al. (2008) An fMRI study of acupuncture using independent component analysis. Neurosci Lett.

149. Bai L, Tian J, Qin W, Pan X, Yang L, et al. (2007) Exploratory analysis of functional connectivity network in acupuncture study by a graph theory mode. Conf Proc IEEE Eng Med Biol Soc 2007: 2023-2026.

150. Qin W, Tian J, Bai L, Pan X, Yang L, et al. (2008) FMRI Connectivity Analysis of Acupuncture Effects on an Amygdala-Associated Brain Network. Mol Pain 4: 55.

151. Qin W, Tian J, Pan X, Yang L, Zhen Z (2006) The correlated network of acupuncture effect: a functional connectivity study. Conf Proc IEEE Eng Med Biol Soc 1: 480-483.

152. Liu P, Oin W, Zhang Y, Tian J, Bai L, et al. (2009) Combining spatial and temporal information to explore function-guide action of acupuncture using fMRI. J Magn Reson Imaging 30: 41-46.

153. Liu P, Zhang Y, Zhou G, Yuan K, Qin W, et al. (2009) Partial correlation investigation on the default mode network involved in acupuncture: an fMRI study. Neurosci Lett 462: 183-187.

154. Yeo S, Kim Y, Choe I-h, Rheu C-h, Choi Y-g, et al. (2009) Reproducibility Between two physicians of fMRI study on the Brain Activity Induced by Acupuncture. Journal of Meridian \& Acupoint 26: 39-51.

155. Raichle ME, Mintun MA (2006) Brain work and brain imaging. Annu Rev Neurosci 29: 449-476.

156. Poldrack RA, Fletcher PC, Henson RN, Worsley KJ, Brett M, et al. (2008) Guidelines for reporting an fMRI study. Neuroimage 40: 409-414.

157. Sun G (2008) Basic Theroy of Traditional Chinese Medicine. Beijing: China Press of Traditional Chinese Medicine. 315 p.

158. Han JS (2011) Acupuncture analgesia: areas of consensus and controversy. Pain 152: S41-48.

159. Dhond RP, Kettner N, Napadow V (2007) Do the neural correlates of acupuncture and placebo effects differ? Pain 128: 8-12.

160. Li X, Liu X, Liang F (2008) Functional brain imaging studies on specificity of meridian and acupoints. Neural Regen Res 3: 777-781.

161. Campbell A (2006) Point specificity of acupuncture in the light of recent clinical and imaging studies. Acupunct Med 24: 118-122.

162. Beissner F, Henke C (2009) Methodological Problems in fMRI Studies on Acupuncture: A Critical Review With Special Emphasis on Visual and Auditory Cortex Activations. Evid Based Complement Alternat Med.

163. Cho ZH, Hwang SC, Wong EK, Son YD, Kang CK, et al. (2006) Neural substrates, experimental evidences and functional hypothesis of acupuncture mechanisms. Acta Neurol Scand 113: 370-377.

164. Chae Y, Park HJ, Hahm DH, Hong M, Ha E, et al. (2007) fMRI review on brain responses to acupuncture: the limitations and possibilities in traditional Korean acupuncture. Neurol Res 29 Suppl 1: S42-48.

165. Zhao L, You ZL, Tang Y, Liang FR (2009) Observation of brain response to acupuncture stimuli using fMRI. Lishizhen medicine and materia medica research 20: 1343-1345.

166. Li XT, Song XG (2009) An overview of the application of functional MRI technique on clinical acupuncture. Journal of Anhui TCM College 4: 78-80.

167. Jiang C, Zhou SY, Zhao L, Li Y (2010) To evaluate the application of fMRI technique in acupuncture research. Journal of practical traditional Chinese medicine 26: 275-277.

168. $\mathrm{Hu} \mathrm{T}, \mathrm{Hu} \mathrm{KM}$ (2009) The application progress on brain response to acupuncture using functional MRI. Med J West China 21: 1987-1988. 